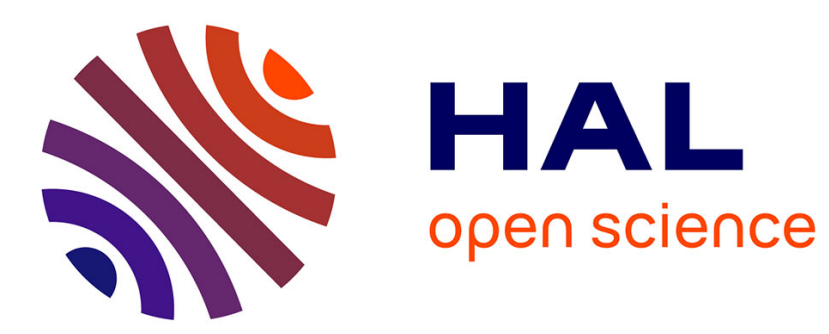

\title{
Relations médecine - sciences dans l'individualisation des maladies nerveuses à la Salpêtrière à la fin du XIXe siècle \\ Jean-Gaël Barbara
}

\section{To cite this version:}

Jean-Gaël Barbara. Relations médecine - sciences dans l'individualisation des maladies nerveuses à la Salpêtrière à la fin du XIXe siècle. Revue d'Histoire des Sciences, 2010. halshs-03090795

\section{HAL Id: halshs-03090795 \\ https://shs.hal.science/halshs-03090795}

Submitted on 30 Dec 2020

HAL is a multi-disciplinary open access archive for the deposit and dissemination of scientific research documents, whether they are published or not. The documents may come from teaching and research institutions in France or abroad, or from public or private research centers.
L'archive ouverte pluridisciplinaire HAL, est destinée au dépôt et à la diffusion de documents scientifiques de niveau recherche, publiés ou non, émanant des établissements d'enseignement et de recherche français ou étrangers, des laboratoires publics ou privés. 


\title{
Relations médecine - sciences dans I'individualisation des maladies nerveuses à la Salpêtrière à la fin du XIX ${ }^{\mathrm{e}}$ siècle \\ Jean-Gaël BARBARA *
}

\begin{abstract}
Résumé : Les recherches cliniques et anatomopathologiques de la seconde moitié du XIX $X^{\mathrm{e}}$ siècle, menées à la Salpêtrière, créent de nouveaux modes d'interaction entre pratiques, théories scientifiques et recherche médicale, dans le domaine spécifique de la nosographie et de la pathogénie des maladies du système nerveux. Nous souhaitons comprendre comment l'essor de la microscopie, I'adoption de la théorie cellulaire, la pathologie expérimentale, les progrès de l'histologie, la théorie du neurone ont pu interagir, en s'associant étroitement à une clinique toujours autonome, par des légitimations, des emprunts et des remises en cause aux bénéfices réciproques.
\end{abstract}

Mots-clés : maladies nerveuses ; étiologie ; causalité ; épistémologie de la médecine ; cadres nosographiques ; théorie du neurone.

Summary : In the second half of the 19th century, at the Salpêtrière hospital in Paris, clinical and anatomopathological researches established new interactions between practices, scientific theories and medical research, in the specific domains of nosography and pathogeny of the diseases of the nervous system. We seek to understand how the rise of microscopy, the acceptance of cell theory, experimental pathology, progress in histology, and the neuron doctrine, could combine with the autonomous field of the clinic and interact through a mutually beneficial process of legitimization, exchange and constructive criticism.

Keywords: nervous diseases; aetiology; causality; philosophy of medicine; nosological frameworks; neuron doctrine.

\footnotetext{
* Jean-Gaël Barbara, Université Pierre-et-Marie-Curie - Paris-6, Case 14, Laboratoire de neurobiologie des processus adaptatifs (UMR 7102 du CNRS), 7, quai Saint-Bernard, 75005 Paris, et chercheur associé au laboratoire SPHERE (UMR 7219 du CNRS), Université DenisDiderot - Paris 7.

E-mail : jean-gael.barbara@snv.jussieu.fr
} 
Jean-Gaël BARBARA

"Comment donc se forme une science médicale? Vous êtes-vous jamais demandé, Messieurs, comment, du fouillis inextricable des faits non classés, avaient pu sortir des cadres nosologiques définis, des affections cliniquement, anatomiquement, voire pathogéniquement déterminées ${ }^{1}$ ?

\section{Introduction}

Le domaine particulier de l'histoire des sciences anatomiques, physiologiques et cliniques du système nerveux est propice pour une étude des relations croisées entre médecine et sciences, aussi bien sur le plan des institutions, des évolutions disciplinaires, des pratiques médicales, que sur un mode épistémologique, en relation avec l'histoire sociale.

$C^{\prime}$ est une telle étude que nous souhaitons tenter ici par l'analyse des modes de constitution des maladies du système nerveux et de l'évolution des cadres nosologiques associés, par la clinique, I'anatomie pathologique, la pathologie expérimentale, I'anatomie normale et la physiologie. On traitera le cas du développement de la neurologie à la Salpêtrière - I'hospice La Vieillesse-Femmes et ses quartiers d'aliénées -, à Paris, entre les années 1850 et le début des années 1900. Nous déborderons par moments ce cadre en considérant certains travaux réalisés dans d'autres lieux par des médecins ayant débuté et/ou terminé leur carrière à la Salpêtrière.

Si nous considérons d'emblée la fin de la période analysée, Jules Dejerine (1849-1917) fait ce constat, le vendredi 31 mars 1911, lors de la leçon inaugurale à la chaire de clinique des maladies du système nerveux qu'il donne à l'hospice de la Salpêtrière ${ }^{2}$ :

" [Si] le clinicien de 1850 revenait parmi nous, la terminologie scientifique que nous employons aujourd'hui serait pour lui à peu près vide de sens. Il reconnaîtrait l'hémorragie, et le ramollissement, les vices de conformation et les traumatismes, comprendrait la signification sémiologique d'un certain nombre de termes par nous usités. Mais les cadres nosologiques, les affec-

1 - Jules Dejerine, Clinique des maladies du système nerveux, Leçon inaugurale, La Presse médicale, 26 (1911), 3-46.

2 - Second successeur à la chaire de Jean-Martin Charcot (1825-1893) de clinique des maladies du système nerveux après Fulgence Raymond (1844-1910). 
Relations médecine - sciences...

tions définies et, à l'heure actuelle, classiques, il les ignorerait à peu près complètement ${ }^{3}$. $"$

Cette citation résume notre question initiale dans le lieu même et l'exacte période où nous la situons. Comment peut-on expliquer une si grande évolution en considérant les rapports médecine-sciences ${ }^{4}$ ?

Pour répondre à cette interrogation, l'école de la Salpêtrière présente les avantages d'avoir été étudiée avec minutie selon le point de vue de I'histoire de la neurologie ${ }^{5}$, de I'histoire de la psychiatrie $^{6}$, et des institutions hospitalières ${ }^{7}$. Mais aussi, d'avoir fait l'objet d'analyses sur les spécificités relatives de ses origines parisiennes, lors de la Révolution française ${ }^{8}$.

Un autre intérêt de la période envisagée est de comprendre l'évolution du cadre philosophique de ce milieu, qui s'enracine dans une vision comtienne classique de la médecine, telle qu'elle se rencontre au début des années 1850 chez les cliniciens membres de la positiviste Société de Biologie, mais qui évolue ensuite vers une définition paradoxale de la pathologie, à la fois comme un domaine plus autonome et en contact plus étroit avec les sciences anatomiques et physiologiques, selon une vision redécouverte chez Auguste Comte (1798-1857) par Jean-François Braunstein ${ }^{9}$.

3 - Dejerine, op. cit. in n. 1, 17.

4 - À cette question, Dejerine répond par la liste des aires de progrès scientifique utiles à la médecine sans entrer dans l'analyse des rapports médecine-sciences.

5 - Alain Lellouch, Jean-Martin Charcot et les origines de la gériatrie (Paris: Payot, 1992) ; Christopher G. Goetz, Michel Bonduelle, Toby Gelfand, Charcot: Constructing neurology (Oxford: Oxford University Press, 1995).

6 - Pierre-Henri Castel, La Querelle de I'hystérie (Paris: Presses universitaires de France, 1998). Serge Nicolas, L'école de la Salpêtrière en 1885, Psychologie et histoire, 1 (2000), 165-207.

7 - Mark S. Micale, The Salpêtrière in the age of Charcot : An institutional perspective on medical history in the late nineteenth century, Journal of contemporary history, 20 (1985), 703-731.

8 - Pour les aspects spécifiques dus à la situation politique de Paris, voir Dora B. Weiner et Michael J. Sauter, The city of Paris and the rise of clinical medicine, Osiris, 18 (2003), 23-42. Pour la critique des spécificités de l'école parisienne, voir Caroline Hannaway et Ann Le Berge, Constructing Paris médicine (Amsterdam : Éditions Rodopi B. V., 1998). Cette connaissance historique détaillée permet de prendre en considération certains thèmes maintenant classiques de I'histoire sociale, les effets dans la clinique du contexte sociopolitique, de l'introduction du laboratoire et de l'adoption des sciences expérimentales.

9 - Jean-François Braunstein, Auguste Comte et la philosophie de la médecine, in Annie Petit, Auguste Comte: Trajectoires positivistes, 1798-1998 (Paris : L'Harmat$\tan , 2003)$. 
Jean-Gaël BARBARA

Nos analyses se concentreront sur trois phases particulières du développement de la neurologie à la Salpêtrière, en insistant sur les aspects épistémologiques qui se déclinent en parallèle des évolutions sociales permettant un rapprochement entre la médecine et les sciences à cette période. Une première phase est constituée par les travaux de deux amis et collaborateurs, Jean-Martin Charcot (1825-1893) et Edmé-Félix-Alfred Vulpian (1826-1887), depuis la fin des années 1850 jusqu'en $1872^{10}$. Il s'agit d'une période de construction active de la neurologie dans le cercle de Charcot, alors que Vulpian s'oriente progressivement vers la pathologie expérimentale.

Le début des années 1870 marque une seconde phase s'étendant jusqu'aux années 1880, avec le grand développement des techniques histologiques des fibres nerveuses et leurs utilisations fructueuses en pathologie. Une importance grandissante est accordée à la pathologie expérimentale de Vulpian et de Dejerine, par une approche qui promeut de nouveaux modèles pathogéniques (recherches des mécanismes pathologiques). Cette période s'achève par des polémiques, souvent vives, au sujet d'étiologies (recherches des causes proximales et distantes des maladies) considérées comme contradictoires - maladies infectieuses et maladies héréditaires - parce que relevant de rationalités en apparence opposées.

Les années 1890 forment une troisième période au cours de laquelle des cadres théoriques se mettent en place, ou se développent plus rapidement que précédemment, la théorie du neurone, les localisations cérébrales et les théories psychothérapeutiques. Nous ne traiterons que du premier de ces cadres constitué par le développement de la théorie du neurone et sa formulation en 1891, qui marque un tournant d'abord discret mais décisif en neurologie. Nous aurons à considérer à ce propos les enjeux épistémologiques de la rencontre des neurologues - comme le premier successeur de Charcot, Fulgence Raymond (1844-1910) - avec les travaux de

10 - C'est l'époque pendant laquelle ils fréquentent ensemble la Salpêtrière. En 1872, Vulpian cède sa chaire d'anatomie pathologique à Charcot, pour occuper la chaire de pathologie expérimentale et comparée de la faculté de médecine de Paris, laissée vacante par la démission de Charles-Édouard Brown-Séquard. 
Relations médecine - sciences...

Santiago Ramón y Cajal (1852-1934 $\left.{ }^{11}\right)$, mais aussi à apprécier I'appui qu'ont pu constituer certaines études de pathologie en faveur de la théorie du neurone.

\section{Clinique et nouvelle rationalité histologique (1857-1870)}

\section{Évolutions de la clinique en dehors des cadres théoriques médicaux et scientifiques}

Lorsqu'en 1853, Charcot et Vulpian deviennent docteurs en médecine, I'aphorisme de Rudolf Virchow (1821-1902) "Omnis cellula e cellula " n'est pas énoncé, mais sa pathologie cellulaire est en ébauche autour d'un concept de cellule pensée comme unité nutritive susceptible de dégénérer, lorsque les conditions physiologiques externes ne lui sont plus favorables ${ }^{12}$. Claude Bernard (1813-1878) et Virchow défendent alors une vision physiologique de la maladie, dans le cadre d'une médecine fondée sur une nouvelle rationalité, la théorie cellulaire pour Virchow, les régulations physiologiques pour Bernard. Cette vision, que Canguilhem a rapprochée des idées de François-Joseph-Victor Broussais (17721838), a été reprise par Comte ${ }^{13}$ et vivement critiquée par Georges Canguilhem (1904-1995). Cependant, ces nouveaux cadres théoriques $\mathrm{n}^{\prime}$ ont pas encore imprégné en profondeur les nosographies.

Canguilhem s'est autorisé à reprendre sur ce point Bernard et Virchow, en pointant un " oubli professionnel ${ }^{14}$ » quant à la place prééminente que doit tenir, selon lui, la clinique par rapport à la physiologie. Pourtant, on sait que Bernard a refusé clairement la subordination de la pathologie à la physiologie, la complexité des maladies ne pouvant pas trouver tous ses déter-

11 - Pour une première approche des travaux de Cajal, voir les articles récents : Edward Jones, Neuroanatomy : Cajal and after Cajal, Brain research reviews, 55 (2007), 248255 ; Juan De Carlos, José Borrell, A historical reflection of the contributions of Cajal and Golgi to the foundations of neuroscience, ibid., 8-16; Javier DeFelipe, Brain plasticity and mental processes: Cajal again, Nature Reviews Neuroscience, 7 (2006), 811-817.

12 - Rudolf Virchow, Ernährungseinheiten und Krankheitsheerde, Virchow's Archiv, 4 (1852), 375, cité in François Duchesneau, Genèse de la théorie cellulaire (Montréal : Bellarmin - Paris : Vrin, 1987), 298.

13 - Georges Canguilhem, Le Normal et le pathologique (Paris: PUF, 1966), 138-142.

14 - Ibid., 139. 
minismes dans l'explication physiologique ${ }^{15}$. En conséquence un médecin comme Charcot n'ayant pas omis de lire et citer les " paroles excellentes [...] et absolument significatives » de Bernard sur ce point ${ }^{16}$-, la pathologie demeure pour le médecin une entité clinique autonome définie essentiellement par ses symptômes et ses signes, selon une perspective générale particulièrement apparente dans I'œuvre de Charcot, et qui domine toute son école. Selon Dejerine, "la première étape [de la neurologie] [...] est une étape de simple classification, où les faits observés se groupent suivant leurs caractères extérieurs ${ }^{17}$ ». Or, " première " ici ne doit pas être compris dans un sens positiviste, mais bien comme un point de départ à ne pas omettre.

Cette méthode a trouvé une justification dans la mise à l'écart positiviste des doctrines médicales, et la valorisation des observations comme faits établis. Aussi s'agit-il en apparence d'un point de décrochement entre une médecine d'observation et ses dimensions théoriques. Mais du point de vue historique, cette clinique n'est pas sans lien avec une heuristique idéaliste plus ancienne se fondant sur l'étude de l'évolution des symptômes (la «marche de la maladie»), le concept de "maladie naturelle », et les principes des classifications, ni avec l'émergence des nouveaux cadres théoriques anatomiques et physiologiques des années 1850.

Pour comprendre la place de cette ancienne heuristique dans les conceptions de Charcot et de son école, rapprochons deux citations respectivement des années 1857 et $1887^{18}$. Selon la première, les "irrégularités morphologiques» de la maladie sont un "centre d'où émanent [...] à distance des caractères spéciaux [...] subordonnés [...] au degré de la difformité et qui la traduisent selon des règles fixes et immuables». Dans la seconde, au sujet de l'hystérie, Charcot dit avoir groupé autour d'un "type complet», "les variétés résultant de la prédominance ou de l'atténuation de plusieurs périodes qui la 15 - Claude Bernard, Introduction à l'étude de la médecine expérimentale (Paris : Flammarion, 1984), 280-282.

16 - Jean-Martin Charcot, Leçons sur les maladies du système nerveux faites à la Salpêtrière (Paris : Delahaye, 1887), t. III, 9. Voir aussi : Pierre-Henri Castel, La Querelle de l'hystérie (Paris : PUF, 1998), 40.

17 - Dejerine, op. cit. in n. 1.

18 - Ces citations sont extraites d'Alain Lellouch, op. cit. in n. 5, 26. 
Relations médecine - sciences...

composent ». De l'une à l'autre de ces deux citations, Charcot n'a pas abandonné la recherche d'une forme pathologique générale, d'où dérivent des variantes, dans l'étude d'affections organiques ou psychiques, ce qui lui vaut, de la part de Sigmund Freud (1856-1939 ${ }^{19}$ ), des critiques reprises et élargies dans un essai de Michel Foucault ${ }^{20}$ : une telle histoire naturelle des maladies organiques n'est pas concevable dans l'étude des maladies mentales. Charcot recherche en effet à cette époque une explication neurologique de l'hystérie et déploie le même type de recherches que pour les maladies nerveuses. Cet archaïsme apparent trahit à la fois une méthode clinique ancienne indispensable, base de toute médecine scientifique selon Dejerine, mais aussi une conception qu'on retrouve détaillée chez Jean-Pierre Falret (1794-1870 ${ }^{21}$ ), revendiquant l'étude de la maladie psychique par la prise en compte de la marche des symptômes, selon les variétés pathologiques, dans le but de les individualiser en tant qu'entités naturelles ${ }^{22}$. Les allusions de Falret à la botanique, et à Antoine-Laurent de Jussieu (1748-1836) en particulier ${ }^{23}$, laissent entendre qu'une maladie naturelle n'est isolable qu'au prix d'une recherche abordant ses différents aspects, selon certains préceptes qui se retrouvent clairement chez des auteurs postérieurs, comme chez Dejerine, Alois Alzheimer (1864-1915 ${ }^{24}$ ), Emil Kraepelin (1856-1926) ou le psychiatre praguois Oskar Fisher ${ }^{25}$.

Dans toutes ces conceptions, le diagnostic différentiel comprend une analyse rendant compte des différents caractères de la maladie et de son évolution. Pour Falret, la perspective, prenant comme modèle Jussieu, vise à unir les données de I'anatomie et de la physiologie, selon les idées de Xavier Bichat (1771-1802), mais aussi celles de la clinique et de la psychologie, par une approche croisée des disciplines que revendique également

19 - Daphne de Marneffe, The construction of clinical knowledge in Charcot and Freud, Signs, 17 (1991), 71-111.

20 - Michel Foucault, Maladie mentale et psychologie (Paris : PUF, 1954).

21 - Castel, op. cit. in n. 16, 20.

22 - Jean-Pierre Falret, Des maladies mentales et des asiles d'aliénés : Leçons cliniques et considérations générales (Paris: Baillière et fils, 1864), xxvii.

23 - Falret, op. cit. in n. 22, xxxiii.

24 - Fabrice Gzil, "Problèmes philosophiques soulevés par la maladie d'Alzheimer », thèse de doctorat (univ. Paris 1, 2007), 146-149. Id., Problèmes philosophiques soulevés par la maladie d'Alzheimer (Paris: PUF, 2009).

25 - Gzil, op. cit. in n. 24 (2009), 46. 
Charcot $^{26}$. Cependant, Dejerine pointe les limites de cette ancienne méthode : "La science [...] groupe les faits par analogie d'abord, par analyse ensuite. Chaque procédé d'examen clinique ou anatomo-pathologique devient le point de départ de différenciations nouvelles ${ }^{27}$ "; en effet, "si ce travail se poursuivait indéfiniment la science médicale $\mathrm{n}^{\prime}$ en arriverait à être formée que de faits particuliers qu'isolent les uns des autres tels ou tels caractères cliniques ${ }^{28} »$. La perspective ancienne de constitution de la maladie est donc toujours opérative à la fin du XIX $X^{\mathrm{e}}$ siècle, mais I'analyse typologique et la recherche multidisciplinaire des causes morbides se situent désormais dans le laboratoire. Cependant, le seuil du changement le plus significatif n'est pas, semblet-il, dans le rassemblement sommaire, dans un réduit, de quelques instruments scientifiques. Dejerine appelle en effet à une nouvelle rationalité et revendique dans ce but, pour la clinique, une nouvelle visée, encore placée au second plan dans les recherches de Charcot des années 1850, qui émerge dans les travaux de Virchow, et qui pourtant était déjà attendue par Falret dans la réforme de la nosographie ${ }^{29}$ : c'est la pathogénie ou la recherche des mécanismes pathologiques - impliquant des causes premières - des maladies qui se développe dans le cadre des nouvelles pratiques microscopiques ${ }^{30}$, à partir de 1862, dans le cercle de Charcot et Vulpian. Or, c'est par la recherche expérimentale des causes des maladies que la clinique va progressivement tisser des liens plus étroits avec les théories scientifiques et leurs pratiques corrélatives. L'interaction de la médecine avec les sciences naît donc aussi d'une évolution de la manière de penser les causes des maladies, par le renouveau de l'école de Paris dans la double perspective de l'école de Giovanni Battista Morgagni (1682-1771) et de la théorie cellulaire.

\section{La pathogénie cellulaire de Virchow à Charcot}

Si la pathogénie n'a pas tenu une place principale dans les premiers travaux de Charcot, elle n'en est pas cependant absente, ne serait-ce qu'en raison de ses études faites dans le sillage direct des travaux de Virchow. Nous prenons pour exemple la

26 - Charcot, op. cit. in n. 16, 20-21.

27 - Dejerine, op. cit. in n. 1.

28 - Dejerine, op. cit. in n. 1.

29 - Falret, op. cit. in n. 22, xxxii.

30 - Les nouveaux aspects des recherches microscopiques sont ceux décrits dans l'ouvrage de Louis Ranvier, Traité technique d'histologie (Paris : Savy, 1875). 
Relations médecine - sciences...

réforme de la pathogénie de l'inflammation par ce dernier. Quels liens entretient-elle avec la pathologie cellulaire dans ses dimensions théoriques et quellesquesnt ésions des fibres nerveuses, au début pas encore de cadre permettant de questionner les liens entre ces deux types de en sont les conséquences dans la nosographie des maladies du système nerveux, dans le contexte initial de Virchow, puis à la Salpêtrière?

Selon une analyse de Duchesneau, Virchow renverse le modèle pathogénique de l'inflammation de Jean Cruveilhier (1791-1874). L'inflammation qui suit une obstruction vasculaire a pour cause, selon lui, une phlébite (inflammation des veines ${ }^{31}$ ). Selon Virchow, le thrombus est considéré comme une formation de corpuscules fibrineux, inductible expérimentalement par injection $\mathrm{d}^{\prime}$ acide dans un vaisseau, déterminant une inflammation des parois vasculaires, avec formation de cellules inflammatoires épithéliales. L'inflammation n'est donc, dans ce schéma, ni la cause, ni la maladie elle-même, mais l'effet d'une obstruction vasculaire. Virchow, qui accorde toute leur importance aux conditions physiologiques nouvelles de l'état pathologique, en appelle à l'étude de la pathologie expérimentale, aidée par la microscopie, dans le contexte de la théorie cellulaire. La maladie semble susceptible d'une recréation par modification de ses facteurs déterminants dans les conditions pathologiques.

Dans le domaine des maladies du système nerveux, $c^{\prime}$ est surtout I'encéphalite qui disparaît en tant qu'entité nosologique, principalement du fait de son remplacement par la pathogénie de I'oblitération vasculaire (Virchow ${ }^{32}$ ). Ce succès, parmi d'autres, a contribué à faire accepter rapidement la pathologie cellulaire en inspirant chez les médecins certains travaux, dont ceux de Charcot: les études concernant les altérations du sang (granules, corpuscules, cristaux), celles sur les embolies par recherche de dépôts fibrineux et sur la claudication intermittente suite à I'oblitération des artères iliaques ${ }^{33}$. Dans certaines études,

31 - Duchesneau, op. cit. in n. 12, 294.

32 - Dejerine, op. cit. in n. 1.

33 - Jean-Martin Charcot, Sur la claudication intermittente observée dans un cas d'oblitération complète de l'une des artères iliaques primitives, Comptes rendus de la Société de Biologie (1858), 225-237. Les travaux de pathologie cellulaire de Charcot de cette période s'intéressent aussi à la structure du cartilage dans la goutte ou encore les affections cellulaires des pathologies du foie. 
Jean-Gaël BARBARA

Charcot critique même Virchow et renverse son modèle pathogénique en attribuant à la paroi vasculaire pathologique un rôle dans la formation du thrombus ${ }^{34}$. Ces travaux témoignent de l'établissement en France de la microscopie dans les hôpitaux ${ }^{35}$, du rôle de la pathologie dans l'essor de la théorie cellulaire et de l'usage médical du microscope ${ }^{36}$. Aussi peut-on dire que, pendant les années 1850 en France, la théorie cellulaire a permis le développement de nouveaux modèles pathogéniques, et qu'en retour, le succès des réformes nosologiques a permis I'extension des cadres théoriques et des pratiques associées dans divers milieux médicaux.

\section{Le modèle pathogénique wallérien et la collaboration Charcot-Vulpian (1862-1875)}

En 1862, Charcot et Vulpian sont nommés chefs de service à la Salpêtrière. Ils démarrent leur collaboration par l'inventaire des patientes de la Vieillesse-Femmes pour leurs études futures. Les conditions de vie de ces femmes sont souvent critiquées par les médecins qui, à l'instar de Charcot, comprennent qu'elles influencent directement leur santé. En même temps, par leurs maladies individuelles, ces patientes constituent une sorte de collection d'histoire naturelle, un "musée anatomopathologique ${ }^{37}$ ". C'est à ce titre un matériau de recherche riche, mais négligé et laissé à de jeunes médecins. Ce sont ces conditions qui déterminent largement le choix de Charcot et Vulpian d'étudier la pathogénie des maladies chroniques de ces femmes. Elles les orientent particulièrement vers l'étude de leurs maladies nerveuses. Mais les travaux antérieurs de Vulpian le portent aussi naturellement à $s^{\prime} y$ intéresser, après sa thèse sur I'origine des nerfs crâniens ${ }^{38}$, et ses études de pathologie expérimentale sur la compression, la section, la dégénérescence et la régénération des nerfs, réalisées avant 1860, dans le labora-

34 - D’après un Exposé des titres et travaux de Charcot cité in Lellouch, op. cit. in n. 5, 183.

35 - Ann La Berge, Medical microscopy in Paris, 1830-1855, in Ann La Berge, Mordechaï Feingold, French medical culture in the XIxth century (Amsterdam : Rodopi, 1994).

36 - Ann La Berge, Debate as scientific practice in nineteenth-century Paris: The controversy over the microscope, Perspectives on science : Historical, philosophical, social, 12 (2004), 424-453.

37 - Charcot, op. cit. in n. 16, 5.

38 - Edmé-Félix-Alfred Vulpian, "Essai sur l'origine de plusieurs nerfs crâniens », thèse de doctorat (faculté de médecine de Paris, 1853). 
Relations médecine - sciences...

toire de son maître Jean-Pierre-Marie Flourens (1794-1867) au Muséum d'histoire naturelle.

C'est à partir de 1862 que les travaux communs de Charcot et Vulpian associent davantage l'histologie du système nerveux aux autopsies réalisées par ou sous la direction de Vulpian. L'histologie demeure encore à cette époque un ensemble de pratiques peu sûres, qui n'ont abouti qu'à des observations isolées, comme la fibre primitive de Remak, ou les cellules nerveuses observées par exemple par Jan Evangelista Purkinje (17871869), dans le cervelet. Mais les travaux d'Augustus Volney Waller (1816-1870), entamés à partir de la fin des années 1840, sur la dégénérescence des nerfs après section, constituent bientôt un puissant cadre physiologique - lequel suscitera des discussions théoriques sur les phénomènes de régénération nerveuse et, beaucoup plus tardivement, au sujet du concept de neurone - mais aussi un cadre pathologique fondamental suscitant enthousiasme et critiques, notamment de la part de Charcot et Vulpian.

Waller est un microscopiste qui débute sa carrière dans le cercle d'Alfred Donné (1801-1878), I'un des premiers partisans parisiens de la microscopie pathologique. Son modèle de la langue de grenouille possède la transparence requise pour observer les modifications morphologiques de ses différents éléments cellulaires après section. Waller décrit en particulier la dégénérescence des fibres nerveuses séparées de leurs cellules et tire la conséquence, à partir de 1852, que le suivi méthodique de la dégénérescence $d^{\prime}$ un nerf après différentes sections permet de découvrir son trajet jusqu'à son centre d'origine, où se localisent les corps cellulaires ${ }^{39}$. Dans le domaine pathologique, les dégénérescences observées après autopsie par I'histologie permettent également en principe de découvrir le centre lésé. Toutes les discussions futures qui entreront dans ce cadre tourneront autour d'une question : un foyer de dégénérescence nerveux (secondaire) est-il toujours associé à une lésion centrale, ou peut-il constituer lui-même une lésion primitive? Une fois encore, un nouveau cadre pathogénique émerge de la phy-

39 - Alan H. Sykes, Servants of medicine, Augustus Waller - father and son - physiologists (York : Ebor Press, 2004). 
Jean-Gaël BARBARA

siologie par une approche de pathologie expérimentale. Mais peut-il constituer une loi générale de la pathologie?

Le concept de dégénérescence secondaire du système nerveux central n'est pourtant pas neuf. Certaines données cliniques du $\mathrm{XVIII}{ }^{\mathrm{e}}$ siècle suggèrent que des atteintes des nerfs surviennent de manière consécutive à une apoplexie et un ramollissement $d^{\prime}$ une région cérébrale. La pathologie cellulaire s'y est également intéressée en reconnaissant au sein des foyers de dégénérescence certains " corpuscules amyloïdes », décrits par Virchow et observés en 1859 par Jules Luys (1828-1897), médecin qui collabore à cette époque avec Charcot pour I'histologie ${ }^{40}$. Le cadre théorique de Waller associant le concept de dégénérescence secondaire à l'analyse microscopique des tubes nerveux lésés semble donc applicable à la pathologie de son temps.

La Société de Biologie est un lieu particulièrement réceptif pour les travaux de Waller, qui y ont été exposés, et discutés, notamment par les pathologistes. Parmi les membres de la société les plus précoces sur ce thème, Adolphe Gubler (1821-1879) rapporte en 1859 des analyses microscopiques encourageantes qui I'amènent à conclure "qu'on devra, à l'avenir, rechercher ces sortes de lésions secondaires [...] dans tous les cas de maladies prolongées de l'encéphale. Les trajets, ramollis passivement par suite d'une transformation rétrograde comparable à celle du fotus mort dans le sein de la mère, pourront éclairer l'anatomiste sur la direction des faisceaux sensitifs et moteurs à travers les masses encéphaliques ${ }^{41} »$.

Les recherches sur les scléroses de la moelle épinière dans les cordons postérieurs, entamées par Vulpian en collaboration avec Charcot, entrent à cette époque dans ce nouveau cadre d'études ${ }^{42}$. Mais, dès 1862 , un de leurs résultats semble contre-

40 - Jules Luys, Jean-Baptiste Hillairet, Observation de paraplégie, Comptes rendus de la Société de Biologie (1859), 68-70. Jean-Martin Charcot, Jules Luys, Altération lardacée ou cireuse du foie, de la rate et de l'un des reins, ibid., 140-143.

41 - Adolphe Gubler, Note sur l'existence de lésions secondaires des centres nerveux dans le cours des ramollissements inflammatoires, Comptes rendus de la Société de Biologie (1859), 79-81, 80.

42 - Jean-Martin Charcot et Edmé-Félix-Alfred Vulpian, De la paralysie agitante, Gazette hebdomadaire de médecine et de chirurgie, 8 (1861), 765-767, 816-820 et 9 (1862), 54-59. Dans ces études, Charcot sera influencé par I'approche clinique de Guillaume Duchenne de Boulogne (1806-1875). 
Relations médecine - sciences...

dire Waller. Chez une patiente, les racines postérieures de la moelle sont très altérées, sans lésion des ganglions spinaux, alors que dans le modèle expérimental de Waller, la lésion effective de ces ganglions induit une dégénérescence de ces mêmes racines ${ }^{43}$. Les auteurs ne $s^{\prime}$ en trouvent pas troublés et affirment en toute sérénité que "I'opinion émise par $M$. Waller et adoptée par la plupart des physiologistes, trouve un appui dans un [de nos] faits [...] ; mais d'un autre côté elle semblerait être ébranlée par l'autre fait [...]». Selon les opinions épistémologiques des auteurs, qui les portent à réfuter une place centrale de la pathologie expérimentale dans l'élaboration des modèles pathogéniques ${ }^{44}$, une exception à la loi de Waller ne représente pas une erreur possible dans l'observation, mais plutôt la confirmation que l'état pathologique peut suivre ses lois propres. Le nouveau cadre interprétatif de Waller, offert par la physiologie à la pathologie, est donc très tôt remis en cause partiellement. Cependant, il constitue un modèle puissant de pathogénie qui incite à rechercher une lésion primitive et des foyers secondaires de dégénérescence. L'histologie accède à la possibilité de reconstruire l'histoire anatomique des lésions, qu'il convient de placer en regard de I'histoire clinique du patient. C'est à l'intérieur d'un tel cadre que se rencontrent l'histologie et la clinique, les recherches de laboratoire après autopsie et les observations récoltées quotidiennement auprès des patients.

\section{Progrès des techniques histologiques et tournant étiologique dans l'étude des maladies du système nerveux (1870-1890)}

\section{Louis Ranvier et la remise en cause des travaux de Vulpian}

L'association du nom de Vulpian à celui de Charcot dans I'attaque de la pathologie expérimentale est d'un certain point de vue surprenante, car certaines études de Vulpian, antérieures à 1862, relèvent de ce domaine : en particulier l'étude des phénomènes de régénération des nerfs après section, justifiant le remplacement de Charles-Édouard Brown-Séquard (1817-1894) à sa chaire de pathologie expérimentale et comparée. Comme

43 - Jean-Martin Charcot et Edmé-Félix-Alfred Vulpian, Sur deux cas de sclérose des cordons postérieurs de la moelle avec atrophie des racines postérieures (tabes dorsalis), Comptes rendus de la Société de Biologie (1862), 155-173, 173.

44 - Goetz, Bonduelle et Gelfand, op. cit. in n. 5, 71. 
Jean-Gaël BARBARA

Flourens, Brown-Séquard s'est également intéressé à la réunion des deux bouts d'un nerf sectionné ${ }^{45}$. Mais c'est Vulpian qui en établit un véritable modèle, dans lequel des fibres néoformées sont générées aux dépens des fibres lésées. Or ce modèle est entièrement remis en cause par Louis Ranvier (1835-1922), histologiste du cercle de Claude Bernard au Collège de France.

Les études de Ranvier doivent être décrites brièvement ${ }^{46}$ dans leur cheminement intellectuel et leurs principaux développements techniques pour comprendre comment elles forment un nouveau cadre histologique des phénomènes de dégénérescence et de régénération des fibres nerveuses. Ce qui nous permettra d'analyser ensuite comment ce cadre justifie les conceptions de Waller, en faisant de sa loi un principe fondamental, dont la validité s'étend à la pathologie.

Les études minutieuses de Ranvier concernant les détails anatomiques dont Bernard conseillait l'étude pour localiser les fonctions physiologiques, mais aussi celles relevant de la pathologie, aboutissent en 1871 à l'observation de constrictions annulaires ou étranglements (les nœuds de Ranvier) dans la gaine de myéline des fibres nerveuses, par I'utilisation peu courante alors du nitrate d'argent, puis de l'acide osmique, après dissociation manuelle des fibres ${ }^{47}$. Dans la perspective cellulaire de Virchow, Ranvier établit qu'un seul noyau de cellule de Schwann est présent entre deux nœuds. Cette norme devient anatomique et physiologique, la déviation de l'une entraînant celle de l'autre. Elle est en conséquence reconnue également en pathologie si l'on admet le concept bernardien de maladie comme écart au normal ${ }^{48}$. Le modèle de Vulpian est donc inva-

45 - Charles-Édouard Brown-Séquard, Sur les altérations pathologiques qui suivent la section du nerf sciatique, Comptes rendus de la Société de Biologie (1849), 136-137. Id., Cas de régénération complète du nerf sciatique, ibid., 137-138.

46 - Voir Jean-Gaël Barbara, Louis Ranvier (1835-1922) : The contribution of microscopy to physiology and the renewal of French general anatomy, Journal of the history of the neurosciences, 16 (2007), 413-431.

47 - Louis Ranvier, Contributions à I'histologie et à la physiologie des nerfs périphériques, Comptes rendus de l'Académie des sciences [CR Acad. sci. dans la suite], 73 (1871), 1168-1171. Voir Barbara, op. cit. in n. 46.

48 - Après section d'un nerf, lors de la dégénérescence de ses fibres, Ranvier observe une multiplication des noyaux de leur gaine de myéline. Inversement, lors de la régénération, leur nombre redevient normal. Louis Ranvier, De la régénération des nerfs sectionnés, CR Acad. sci., 76 (1873), 491-495. 
Relations médecine - sciences...

lidé ${ }^{49}$, ce qui renforce les conceptions de Waller comme explication de toute dégénérescence secondaire pathologique.

\section{Deux émules de Ranvier et Waller dans l'école de Vulpian : Dejerine et Babinski}

Vulpian est donc contraint en 1873 de changer d'avis, et c'est à I'un de ses élèves, Dejerine, de répondre à l'attaque en expliquant l'erreur de son maître et, si possible, en critiquant Ranvier, ce qu'il réussit finalement assez bien ${ }^{50}$. Pour Dejerine, Vulpian a coloré par le carmin la gaine de Schwann et I'a interprétée par erreur comme la persistance de la fibre nerveuse ${ }^{51}$. Mais ce qui est remarquable, c'est de voir comment dans le domaine pathologique, Dejerine parvient à maîtriser rapidement toutes les techniques de Ranvier, au point non seulement de reproduire ses résultats, mais de l'attaquer sur un point de détail, concernant la corrélation entre la multiplication des noyaux des cellules de Schwann et la perte de fonction du nerf trois jours après section ${ }^{52}$. Dans la conclusion de son étude, Dejerine se livre à un jeu critique, en réfutant I'ancienne conception de Flourens et Vulpian de la dégénérescence du nerf due à l'interruption de sa fonction, et la théorie de Claude Bernard - partagée par Ranvier - d'une régulation nerveuse négative de la nutrition des nerfs. Reste dès lors la théorie wallérienne, qui sort gagnante de cette discussion. En référence à Waller, Dejerine a réussi à mettre Ranvier derrière lui et à placer l'école de Vulpian sur le devant de la scène.

Si Dejerine se fait fort de peu citer Ranvier, il ne peut que louer au détour d'une longue phrase ses "remarquables recherches [qui] ont modifié complètement nos connaissances sur la structure des nerfs ${ }^{53}$ [...] ». Chef de clinique de Charcot de 1885 à 1887, Joseph Babinski (1857-1932) prend un chemin similaire

49 - Jean-Marie Philipeaux et Alfred Vulpian, La régénération des nerfs séparés des centres nerveux, CR Soc Biol (1859), 343-414. Jean-Marie Philipeaux et Alfred Vulpian, Note sur des expériences démontrant que des nerfs séparés des centres nerveux peuvent, après s'être altérés complètement, se régénérer tout en demeurant isolés des centres, et recouvrer leurs propriétés physiologiques, CR Acad. sci., 49 (1859), 507-509.

50 - Jules Dejerine, Recherches sur la dégénérescence des nerfs séparés de leurs centres trophiques, Archives de physiologie (1875), 567-587.

51 - Dejerine, op. cit. in n. 50, 568.

52 - Voir Jean-Gaël Barbara, Louis-Antoine Ranvier (1835-1922), Journal of neurology, 253 (2006), 399-400.

53 - Dejerine, op. cit. in n. 50, 571. 
Jean-Gaël BARBARA

et s'attaque désormais à ce qui apparaît chez Charcot comme une exception à la loi de Waller. En effet, Charcot a décrit dans la sclérose en plaques des lésions nerveuses multiples qui ne s'accompagnent pas d'une dégénérescence antérograde des fibres nerveuses.

"Il existe, note Charcot, $[\ldots]$ un point noir dans nos horizons. Je veux parler d'une exception notoire, qui jusqu'à ce jour du moins, semble contredire la loi (loi wallérienne ${ }^{54}$ ): dans la sclérose en plaques, les lésions, alors même qu'elles sont très étendues, ne produisent pas de dégénérations secondaires. J'ai émis dans le temps l'hypothèse, que cela tient à la persistance plus longue des cylindres axiles dans les foyers de sclérose multiloculaire ${ }^{55}$ [...]. »

Comme précédemment pour Vulpian, les limites techniques n'ont pas permis de voir la réalité, ici le maintien de l'intégrité des axones, ce que démontre Babinski par les techniques de Ranvier ${ }^{56}$. Babinski montre ainsi que le modèle wallérien peut servir de cadre théorique à deux pathologies fondamentales. Dans la sclérose en plaques, la fragmentation de la myéline est comparable à celle du bout central des fibres nerveuses sectionnées, alors que dans la sclérose systématique, les dégénérescences secondaires se rapprochent de celles décrites par Ranvier dans le bout distal. Ce sont en réalité les progrès techniques dans la visualisation des fibres nerveuses et leur maîtrise par des histologistes comme Ranvier qui sont ici déterminants pour faire d'un ensemble de données histologiques précises de pathologie expérimentale un cadre rassemblant et permettant d'interpréter certaines pathologies nerveuses dégénératives.

\section{L'extension du modèle pathogénique wallérien}

L'analyse des travaux de Dejerine de 1875 à 1890 permet de suivre le rôle du modèle pathogénique wallérien dans les recherches consacrées à un ensemble étendu de pathologies du système nerveux. Dans la majeure partie des cas, il s'agit de comprendre la topographie de lésions centrales (cerveau, bulbe,

54 - Parenthèse de Charcot.

55 - Jean-Martin Charcot, Leçons sur les localisations dans les maladies du cerveau et de la moelle épinière (Paris: Delahaye et Lecrosnier, 1876), t. 1, 271.

56 - Joseph Babinski, "Étude anatomique et clinique sur la sclérose en plaques », thèse de doctorat (faculté de médecine de Paris, 1885). 
Relations médecine - sciences...

moelle, ganglions) et nerveuses (racines de la moelle, nerfs périphériques, terminaisons nerveuses de la peau et des muscles), afin de distinguer les lésions primaires des secondaires. Au fil de ses différentes études, les progrès de Dejerine en histologie sont manifestes. Il adopte progressivement les raffinements techniques de Ranvier, précise les durées d'incubation dans les alcools et acides (I'acide osmique surtout). Il réalise des comptages précis des noyaux des cellules de Schwann entre deux nœuds de Ranvier, en adoptant finalement ses normes après une réticence initiale. L'ère de l'inspection macroscopique des coupes est révolue, cette époque selon Dejerine où " on se contentait d'examiner la moelle à l'œil nu, moyen absolument insuffisant, comme on le sait actuellement, et n'ayant aucune valeur scientifique ${ }^{57} »$.

Le rôle du modèle wallérien est évident en pathologie à cette époque. De nombreuses études se perdaient précédemment dans les descriptions de multiples altérations de cellules - dans la tradition de Virchow - ou de fibres nerveuses, sans hypothèse sur leurs relations. Le nouveau modèle pathogénique incite à mieux circonscrire l'étendue des lésions de chaque type, pour en rechercher les liaisons anatomiques et pathologiques. Ainsi Dejerine recherche-til les altérations centrales de la moelle épinière dans son étude sur la malformation congénitale du pied-bot équin :

«Depuis l'époque (1865) où l'existence d'une atrophie cellulaire des cornes antérieures dans la paralysie infantile a été démontrée [...] un certain nombre de faits analogues ont été publiés [...] Ce qui nous a engagé à publier le cas actuel, c'est la limitation exacte de ce processus à la partie antérieure du cordon latéral, sur la limite externe de la corne atrophiée ${ }^{58}$ [...]. »

Les possibilités techniques nouvelles de I'histologie incitent Dejerine à reprendre, en 1876, I'étude de la paralysie ascendante aiguë, un sujet sur lequel il continuera à travailler des années durant et qui formera le sujet de sa thèse de doctorat de $1879^{59}$. Cette pathologie s'apparente aux myélites aiguës,

57 - Jules Dejerine, Notes sur un cas de paralysie ascendante aiguë, Archives de physiologie (1876), 312-317, 315.

58 - Jules Dejerine, Notes sur l'état de la moelle épinière dans un cas de pied-bot équin, Archives de physiologie (1875), 253-256, 256.

59 - Id., "Recherches sur les lésions du système nerveux dans la paralysie ascendante aiguë », thèse de doctorat (faculté de médecine de Paris, 1879). 
Jean-Gaël BARBARA

inflammations démyélinisantes de la moelle, dont on sait à présent qu'elles sont le plus souvent d'origine infectieuse. En 1859, Jean-Baptiste-Octave Landry (1826-1865) en décrit un syndrome apparenté, avec paralysie flasque des membres inférieurs qui envahit progressivement les muscles du tronc, des membres supérieurs et du $\mathrm{cou}^{60}$. L'examen macroscopique ne permet pas de mettre en évidence une lésion de la moelle. S'agit-il dès lors d'une forme de myélite ou d'une autre affection impliquant une dégénérescence des centres nerveux ${ }^{61}$ ? L'existence même de cette pathologie repose sur la recherche d'une pathogénie de type wallérien, ce que résume ainsi Dejerine : "Si I'on vient à trouver des altérations des racines antérieures (de la moelle épinière) dans les différentes régions de la moelle, la paralysie ascendante aiguë sera alors expliquée d'une façon beaucoup plus rationnelle ${ }^{62}$. " L'individualisation d'une pathologie semble dès lors possible par la mise en évidence d'un type nouveau de pathogénie.

Ce que Dejerine recherche donc avant tout, c'est une lésion médullaire, signe d'une dégénérescence centrale qui chemine telle une inflammation jusqu'aux régions supérieures du corps. II déploie toute son énergie, use de la panoplie entière de ses techniques (I'acide osmique, I'acide picrique, le picrocarminate, I'aniline, I'hématoxyline), de l'examen macroscopique et de la microscopie de toute partie susceptible d'être lésée (peau, muscles, nerfs, moelle, méninges, encéphale, etc). En 1878, il parvient à étudier deux nouveaux cas de paralysie ascendante aiguë et découvre des lésions des racines antérieures. Suivant la méthode de Ranvier, il décrit une " fragmentation de la myéline en gouttes et en gouttelettes [...]; [une] hypergenèse du protoplasma de chaque segment inter-annulaire, et une multiplication des noyaux de la gaine de Schwann ${ }^{63}[\ldots]$. » Dans sa thèse, Dejerine fait les mêmes observations et ajoute : " À l'état normal il n'existe qu'un seul noyau dans chaque segment interannulaire (Ranvier ${ }^{64}$ ). » Dejerine croit donc tenir un modèle

60 - Jean-Baptiste Landry, Note sur la paralysie ascendante aiguë, Gazette hebdomadaire de médecine et de chirurgie, 6 (1859), 472-474.

61 - Il s'agit aujourd'hui d'un syndrome rencontré dans la poliomyélite antérieure aiguë, certaines polynévrites, et certaines maladies infectieuses ou parasitaires.

62 - Jules Dejerine, Notes sur un cas de paralysie ascendante aiguë, Archives de physiologie (1876), 312-317, 317.

63 - Id., Sur l'existence de lésions des racines antérieures dans la paralysie ascendante aiguë, CR Acad. sci., 87 (1878), 101-102, 101

64 - Id., op. cit. in n. 59, 20. 
Relations médecine - sciences...

pathogénique sûr qui confirme les données de Waller et Ranvier. Mais André-Victor Cornil (1837-1908), collègue d'internat de Ranvier, médecin histologiste chez Charcot depuis 1863, fait remarquer dans son Manuel d'histologie pathologique ${ }^{65}$ combien les lésions des racines antérieures décrites par Dejerine - qui constituent le cheval de Troie de ses études sur la moelle lui donnant droit d'entrée dans ce domaine de recherche - sont ténues et ne semblent pas pouvoir expliquer entièrement des troubles fonctionnels aussi graves. Cette critique classique pose la question de la pathologie possiblement sine materia, pour laquelle on invoquait un trouble dynamique ou fonctionnel, qu'on pouvait croire seulement sine visibilia, dans l'attente de meilleurs moyens techniques, mais que d'autres expliquaient sans réserve par des anomalies histologiques précises, peu marquées. Dejerine n'aborde d'ailleurs pas le problème, mais il pose ce qui est pour lui la question fatidique : "S'agit-il d'une névrite ou bien $d^{\prime}$ un processus dégénératif purement passif ${ }^{66}$ ? »Sans preuve matérielle directe, Dejerine adopte sans réserve le cadre pathogénique wallérien :

« Il est pour nous très-probable ; quoique nous ne puissions pas en donner une démonstration précise, que les lésions des racines que nous avons constatées à l'examen microscopique, sont secondaires à une altération des cellules des cornes antérieures de la substance grise ${ }^{67}$.

"On sait, depuis les mémorables expériences de M. Waller, que l'intégrité anatomique et fonctionnelle des racines antérieures, est subordonnée entièrement à l'intégrité des cellules des cornes antérieures, qui sont leur véritable centre trophique $[\ldots]$ La portée de ces expériences est énorme, et leurs résultats ont été d'un grand secours dans l'étude de l'anatomie et de la physiologie pathologiques d'un grand nombre d'affections de la moelle épinière $[\ldots]$ Les résultats anatomo-pathologiques, concordent avec les faits expérimentaux, en montrant l'extrême rareté, pour ne pas dire plus, de l'inflammation des nerfs en dehors des cas où, pour une cause ou pour une autre, ils sont soustraits à l'influence de leurs centres trophiques ${ }^{68}[\ldots]$. »

65 - André-Victor Cornil, Louis Ranvier, Manuel d'histologie pathologique (Paris : Baillière, 1869, 1873, 1876)

66 - Dejerine, op. cit. in n. 59, 35

67 - Dejerine, op. cit. in n. 59, 56.

68 - Dejerine, op. cit. in n. 59, 54. 
Jean-Gaël BARBARA

L'affaire peut donc paraître entendue. Mais si l'on a souhaité insister quelque peu sur ce point, $c^{\prime}$ est parce que ce modèle pathogénique est adopté trop tôt de manière systématique, comme Dejerine le reconnaîtra lui-même par la suite. C'est ce que montre la diversité des maladies auxquelles on assigne alors une pathogénie wallérienne. Elles appartiennent à trois groupes; certaines affections cutanées d'origine nerveuse, certaines paralysies et certaines atrophies musculaires. À propos du premier groupe, I'anatomie s'était depuis le début du siècle intéressée aux terminaisons des fibres nerveuses de la peau pour comprendre notamment la physiologie des sensations. Les techniques de Ranvier permettaient de visualiser ces fibres dans la peau et leurs terminaisons. Dejerine recherche, quant à lui, entre 1876 et 1881, des fibres lésées dans des affections comme le pemphigus, la pellagre et les escarres ${ }^{69}$. À chaque fois, il s'étonne de ce que les lésions observées ressemblent à celles du bout périphérique d'un nerf sectionné, et cette analogie l'incite à considérer, parfois sans preuve, que ces lésions cutanées sont trophiques et consécutives à l'altération d'un centre nerveux. II y a là, semble-t-il, une dérive dans l'application du modèle wallérien. Les études de paralysies sont plus claires, car souvent les altérations sont corrélées avec des lésions des cellules des cornes antérieures, ce qui indique une pathogénie wallérienne classique, par lésion des cellules motrices ${ }^{70}$. C'est alors que Dejerine propose une définition de la pathogénie wallérienne: "L'altération des nerfs de cause centrale [doit être] toujours rigoureusement proportionnelle à celle de leurs noyaux d'origine $^{71}$ [...]. » Mais dans une étude de 1883, Dejerine rencontre une complexité qui lui fait énoncer une ancienne idée, déjà utilisée. Une lésion nerveuse peut certes être d'origine centrale, mais certaines surviennent indéniablement à un point quelcon-

69 - Jules Dejerine, Sur l'existence d'altérations des extrémités périphériques des nerfs cutanés, dans un cas d'éruption de bulles de pemphigus, CR Acad. sci., 83 (1876), 281-283. Id., Paralysie générale - troubles trophiques cutanés, pemphigus - lésion de la moelle et des extrémités nerveuses périphériques, Archives de physiologie (1876), 317-323. Id., Sur les altérations des nerfs cutanés dans la pellagre, CR Acad. sci., 93 (1881), 91-92. Id., Sur I'existence d'altérations des nerfs cutanés, dans certaines eschares, survenant dans le cours des maladies du cerveau ou de la moelle épinière, Comptes rendus de la Société de Biologie (1882), 77-78.

70 - Jules Dejerine, Note sur les lésions du système nerveux dans la paralysie diphtérique, CR Acad. sci., 84 (1877), 1110-1112. Id., Recherches sur les lésions du système nerveux dans la paralysie diphtérique, Archives de physiologie (1878), 107-143.

71 - Jules Dejerine, Étude anatomique et clinique sur la paralysie labio-glosso-laryngée, Archives de physiologie (1883), 180-227, 208. 
Relations médecine - sciences...

que de leur parcours, pour une autre raison, sans lésion du centre d'origine ${ }^{72}$. Le modèle wallérien $\mathrm{n}^{\prime}$ est donc pas obligatoire ; il s'agit d'une pathogénie courante, mais pas unique, même si des atrophies musculaires (troisième groupe) peuvent être expliquées par une lésion des cellules des cornes antérieures d'après les travaux de l'école de la Salpêtrière ${ }^{73}$.

\section{Nouvelles exceptions à la pathogénie wallérienne}

Le modèle de la dégénérescence wallérienne rencontre bientôt d'autres faits contradictoires, dont certains sont anciens, ainsi que le rappelle Dejerine, dès 1878 , dans les cas de la section traumatique d'un nerf après amputation d'un membre. Le nerf subit alors des altérations sans lien direct avec une lésion centrale. Et Dejerine se rallie à l'ancienne opinion de son maître, à savoir que le nerf dégénère par défaut $d^{\prime}$ usage ${ }^{74}$.

Nous ne pouvons faire une analyse détaillée des divers travaux de Dejerine qui analysent d'autres lésions primitives de fibres nerveuses rencontrées dans des névrites dites périphériques. Précisons plutôt un rejet net de la pathogénie wallérienne comme modèle unique. En 1890, Dejerine est en mesure de dresser un premier bilan :

"Les travaux de ces dernières années ont montré le rôle considérable joué par la névrite périphérique dans la pathologie nerveuse. La neuro-pathologie moderne est entrée résolument dans la voie de la décentralisation, et cherche actuellement dans des lésions d'ordre périphérique l'explication de certaines affections, regardées pendant trop longtemps comme relevant d'altérations centrales de la moelle épinières ${ }^{75}$. »

Il se fait encore plus catégorique lorsqu'il répond à un mémoire d'Édouard Brissaud (1852-1909) à la séance du 26 juillet 1890 de la Société de Biologie. "Dans la communication de M. Brissaud, dit Dejerine, il y a à la fois une question et une doctrine. [...] II 72 - Dejerine, op. cit. in n. 71, 226.

73 - Jules Dejerine, Atrophie musculaire et paraplégie dans un cas de syphilis maligne précoce, Archives de physiologie (1876), 430-442.

74 - Jules Dejerine, Sur les altérations de la moelle épinière, Comptes rendus de la Société de Biologie (1878), 23-32.

75 - Jules Dejerine, De la nature périphérique de certaines paralysies dites spinales aiguës de l'adulte, Archives de physiologie (1890), 256-261. 
Jean-Gaël BARBARA

n'admet comme vraiment caractéristiques que les lésions présentant les caractères de la dégénérescence dite wallérienne [...] Ici rien ne prouve que le processus soit semblable à celui de la dégénérescence produite expérimentalement ${ }^{76}$. " Des études ultérieures de Dejerine confirment ce point de vue et démontrent $d^{\prime}$ autres exceptions au modèle. Elles donnent aussi une vision plus complète des divers modes de dégénérescences pathologiques, comme les dégénérescences rétrograde ${ }^{77}$ ou ascendante ${ }^{78}$, toutes deux en ordre inverse de la wallérienne antérograde, et les foyers dégénératifs isolés comme la sclérose primitive des cordons latéraux de la moelle ${ }^{79}$.

L'histoire du modèle pathogénique wallérien est donc complexe. Il s'impose malgré quelques premières exceptions qu'on avait cru établir par des techniques insuffisantes. Puis il s'étend à un domaine plus large jusqu'à réformer la nosographie, tout en continuant son dialogue avec des faits anciens, dont certains remettent finalement en cause sa généralité en pathologie. Ce cadre anatomophysiologique, importé dans la pathologie avec toutes ces techniques et dichotomies conceptuelles (entre cellules nerveuses et fibres nerveuses), a été cependant un champ essentiel de recherches et de réflexions incessantes, où l'on voit certaines idées s'épanouir, reculer et revenir en avant sur certaines questions, pour appréhender la complexité des formes pathologiques par le développement d'une nouvelle rationalité scientifique et médicale. En ce sens, ce modèle pathogénique fut le véritable moteur des recherches de pathologie, en permettant son interaction avec I'histologie et la physiologie expérimentales.

\section{Le tournant étiologique des années 1880-1890}

Les discussions autour du modèle de pathogénie wallérienne des années 1870 permettent aux neurologues de la Salpêtrière de

76 - Jules Dejerine, À propos du mémoire de M. Brissaud, Comptes rendus de la Société de Biologie (1890), 506-508.

77 - Jules Dejerine, Sur un cas de dégénérescence rétrograde des fibres pyramidales de la moelle dans les cordons antérieurs et latéraux, Archives de physiologie (1896), 128-139.

78 - Jules Dejerine, Jules Sottas, Sur un cas de dégénérescence ascendante dans les cordons antérieurs et latéraux de la moelle, Comptes rendus de la Société de Biologie (1895), 436-439.

79 - Jules Dejerine, Note sur un cas de paraplégie spasmodique acquise par sclérose primitive des cordons latéraux, Comptes rendus de la Société de Biologie (1895), 776-778. 
Relations médecine - sciences...

mieux réfléchir aux phénomènes morbides impliqués dans la formation des lésions nerveuses qui en dépendent. C'est la recherche de la pathogénie, que Claude Bernard définit comme I'explication de la production des maladies ${ }^{80}$, c'est-à-dire - selon une définition plus récente des années 1930 - l'étude du "mécanisme par lequel les causes morbides déterminent les maladies ${ }^{81}$ ". Mais il peut y avoir un certain éloignement entre les mécanismes morbides et leurs causes. En effet, entre la pathogénie et l'étiologie, se dérobe à l'analyse directe tout l'espace situé entre causes proches et lointaines de la maladie. Mais ce fossé peut être délimité, à un pôle, par certaines corrélations statistiques entre phénomènes distants, et à l'autre, par la possibilité de décrire des causalités directes entre l'apparition d'éléments morbides (les lésions) et certains processus pathogéniques, à I'intérieur de cadres explicatifs précis, à l'instar de ce qui est réalisé pour le modèle pathogénique wallérien.

Avant I'élaboration de modèles pathogéniques précis, le médecin appréhende l'étiologie des maladies par leur degré de proximité avec les causes qui les déterminent. À propos de la folie, Étienne-Jean Georget (1795-1828) différencie en 1820, dans une approche empreinte d'aristotélisme, les causes prédisposantes (hérédité, suites de couche, âge), des causes efficientes directes physiques ou morales, des causes efficientes indirectes physiologiques et pathologiques ${ }^{82}$. Mais à partir des années 1870, on assiste à un certain rapprochement entre les concepts d'étiologie et de pathogénie, qu'il est possible de suivre dans I'œuvre de Charcot. Les étiologies du rhumatisme articulaire et de la goutte, que Charcot décrit autour de 1870, demeurent encore classiques en ce qu'elles comprennent le climat, l'hérédité, I'âge, le sexe, I'humidité, I'alimentation et les liens avec $\mathrm{d}^{\prime}$ autres pathologies ${ }^{83}$. Dans ses leçons sur les maladies du système nerveux, faites au cours des années 1872-1873, Charcot note, au sujet de la pathogénie des attaques apoplectiformes, I'insuffisance de la théorie de la congestion ${ }^{84}$. Mais ce sont les

80 - Claude Bernard, Principes de médecine expérimentale (Paris: PUF, 1947), 297.

81 - Dictionnaire de l'Académie française (Paris: Hachette, 1932-1935), 8 éd.

82 - Étienne-Jean Georget, De la folie, considérations sur cette maladie (Paris : Crevot, 1820), chap. II.

83 - Jean-Martin Charcot, Leçons cliniques sur les maladies des vieillards et les maladies chroniques (Paris: Delahaye, 1874), $8^{\mathrm{e}}$ et $17^{\mathrm{e}}$ leçons.

84 - Jean-Martin Charcot, Leçons sur les maladies du système nerveux faites à la Salpêtrière (Paris : Delahaye, 1872-1873), t. I, $8^{\mathrm{e}}$ leçon. 
analyses histologiques de la sclérose en plaques, constituant la sixième leçon de la même série, qui lui permettent de proposer une véritable pathogénie, ou selon son mot, un « mode de succession des lésions ${ }^{85}$ ». Charcot y propose un schéma explicatif de la dégénérescence des fibres nerveuses : «[...] le processus morbide est en pleine activité : c'est là, en effet, que, comprimé de tous côtés et étouffé [...] le cylindre médullaire [de la fibre nerveuse] s'amoindrit progressivement, puis disparaît ${ }^{86}[\ldots] »$, selon une vision assez mécaniste. Dans sa Clinique des maladies du système nerveux des années 1893, Charcot tente un " essai de pathogénie » de la migraine ophtalmique ${ }^{87}$ et de la sclérose latérale amyotrophique. La pathogénie s'impose donc progressivement à Charcot comme une étude à part, qui s'insère entre l'anatomopathologie et l'étiologie, par la reconnaissance d'un certain déterminisme dans l'évolution des lésions élémentaires constantes, comprises dans leur histoire propre, en lien étroit avec l'histoire clinique du patient.

Ce tournant pathogénique de Charcot témoigne de son intérêt grandissant pour l'étiologie ${ }^{88}$, dans le contexte de sa nouvelle chaire de clinique des maladies du système nerveux, créée pour lui en 1882. Cependant, nous ne pensons pas que Charcot avait un intérêt initialement faible pour l'étiologie. Il en a plutôt une conception classique qui se rapproche progressivement de la pathogénie. D'ailleurs, un moyen d'étude commun remarquable apparaît grâce auquel, dès cette époque, pathogénie et étiologie se rejoignent et coopèrent. C'est l'étude des interactions entre pathologies. Elle participe à la fois de l'étiologie classique (une maladie en favorise une autre) et de l'anatomopathologie (savoir si une lésion peut évoluer en une autre et déterminer une pathologie comme cause directe d'une autre). Dejerine cite ainsi la "doctrine de la téphro-myélite » comme "cause pathogénique unique » de la paralysie atrophique de I'enfance ${ }^{89}$.

85 - Charcot, op. cit. in n. 84, $6^{\mathrm{e}}$ leçon

86 - Charcot, op. cit. in n. 84, 192.

87 - Jean-Martin Charcot, Clinique des maladies du système nerveux, leçons du professeur, mémoires, notes et observations parus pendant les années 1889-90 et 1890-91 (Paris: Babé Delahaye, 1892-1893), 70 et suiv.

88 - Goetz, Bonduelle, Gelfand, op. cit. in n. 5, 258-259.

89 - Jules Dejerine, Contribution à l'étude de la paralysie atrophique de l'enfance à forme hémiplégique (téphro-myélite unilatérale), Archives de physiologie (1888), 375-388, 375-376. 
Relations médecine - sciences...

L'approche pathogénique vise à décrire des ensembles de processus morbides dont certains peuvent être en effet communs à plusieurs pathologies, ou en représenter différents stades d'évolution. Mais en marge de ces processus spécifiques, Charcot a également recours, comme on l'a vu plus haut, à des modèles mécaniques (I'explication de la disparition de la fibre nerveuse par compression du tissu pathologique environnant). Un tel type de modèle se retrouve également chez Ranvier, à la même époque, au sujet de la dégénérescence de la fibre nerveuse après section du nerf ${ }^{90}$. Or, il a déjà été noté que, d'une part, Dejerine critique Ranvier sur ce point en $1875^{91}$ et, d'autre part, que Babinski démontre en 1885 I'erreur du schéma mécanique de la sclérose en plaques de $\operatorname{Charcot}^{92}$. "II ressort, dit Babinski, dans sa thèse de 1885, [...] que la destruction des gaines de myéline loin d'être sous la dépendance d'un phénomène mécanique, d'une compression exercée sur les tubes nerveux par le tissu conjonctif de nouvelle formation, est au contraire liée à un phénomène vital et résulte principalement de l'activité nutritive des cellules de la névroglie et de cellules migratrices ${ }^{93}$. " Cette querelle est en fait significative de l'évolution des modèles de pathogénies, qui fonde une véritable physiologie des processus morbides.

C'est au cours de cette période qu'intervient une autre querelle connexe touchant à l'implication de deux facteurs étiologiques importants dans les maladies du système nerveux, I'hérédité et la maladie infectieuse. L'une et l'autre ont leurs partisans et ces deux étiologies sont alors pensées comme contradictoires. L'œuvre de Charcot abonde en discussions sur le rôle de l'hérédité dans la récurrence de maladies nerveuses au sein de certaines familles. Freud a eu avec lui, sur ce point, une correspondance critique, car Charcot prenait souvent l'exemple en privé des fragilités nerveuses au sein des familles juives, des signes cliniques dont les filiations lui paraissaient évidentes ${ }^{94}$. Dans l'école de la Salpêtrière, il s'agit là d'un thème de longue tradition ${ }^{95}$, repris par exemple par Dejerine dans sa thèse d'agré-

90 - Louis Ranvier, Recherches sur I'histologie et la physiologie des nerfs, Archives de physiologie normale et pathologique, 4 (1872), 427-446.

91 - Dejerine, op. cit. in n. 50.

92 - Babinski, op. cit. in n. 56.

93 - Babinski, op. cit. in n. 56, 24.

94 - Toby Gelfand, Charcot's response to Freud's rebellion, Journal of the history of ideas, 50 (1989), 293-307.

95 - Goetz, Bonduelle, Gelfand, op. cit. in n. 5, xvii, 259. 
gation ${ }^{96}$. Si I'hérédité semble alors tenir une place évidente dans l'étiologie des maladies nerveuses, I'absence de tout consensus sur les mécanismes de transmission des caractères innés empêche certaines doctrines, comme celle du plasma germinatif de Friedrich Leopold August Weismann (1834-1914), adoptée $d^{\prime}$ 'ailleurs par Dejerine, d'entrer en interaction avec les questions de pathogénie. La conception du rôle de l'hérédité demeure en conséquence un domaine empirique de la clinique, où les conceptions épistémologiques a priori demeurent prééminentes.

L'une d'entre elles, peut-être la plus fondamentale, est que les maladies du système nerveux appartiennent à une même famille et ont une origine commune ${ }^{97}$. Cette position explique deux orientations générales, I'adoption du concept de famille névropathique ${ }^{98}$ (le maintien de maladies au fil des générations dans une même famille), dans un contexte de déterminisme social, et le refus de l'étiologie infectieuse. En effet, au sujet de la première, la reconnaissance $d^{\prime}$ une origine commune des maladies nerveuses favorise l'idée qu'une maladie quelconque dans une famille peut être un facteur de développement d'une autre, différente, à la génération suivante. Pour la seconde orientation, l'étiologie infectieuse est souvent rejetée en partie parce qu'on ne comprend pas comment une pluralité de maladies infectieuses peut aboutir à des maladies nerveuses nettement définies par des lésions constantes. C'est ainsi que Dejerine note au sujet de la téphro-myélite de l'enfance qu'une "systématisation aussi rigoureuse de la lésion n'est guère favorable à la doctrine qui voit, dans la téphro-myélite de I'enfance, la localisation spinale d'une maladie infectieuse ${ }^{99}$. „ Ces querelles entre hérédité et étiologie infectieuse ont donné lieu à une véritable crise au congrès international de médecine de $1881^{100}$ et une rupture s'est progressivement dessinée entre Charcot d'une part, représentant d'une tradition orthodoxe favorisant I'hérédité et, d'autre part, Vulpian, Dejerine ${ }^{101}$, Cornil, favorables à ce qui devient progressivement le modèle pathogénique pasteu-

96 - Jules Dejerine, L'Hérédité dans les maladies du système nerveux, thèse d'agrégation (Paris : Asselin et Houzeau, 1886).

97 - Dejerine, op. cit. in n. 96, chap. II.

98 - Gelfand, op. cit. in n. 94, 296.

99 - Dejerine, op. cit. in n. 89, 387.

100 - Goetz, Bonduelle, Gelfand, op. cit. in n. 5, 259-260.

101 - En 1884, Dejerine publie un article de microbiologie : Jules Dejerine, Recherche du bacille de Koch dans la tuberculose calcifiée, Comptes rendus de la Société de Biologie (1884), 500-505. 
Relations médecine - sciences...

rien, sans qu'ils abandonnent d'ailleurs leurs vues sur le rôle étiologique des antécédents familiaux qui, pour certaines pathologies, étaient fondées d'un point de vue génétique.

Ces considérations démontrent assez clairement comment l'étiologie, telle qu'on la comprend au XVIII ${ }^{\mathrm{e}}$ siècle, perd du terrain et se confond progressivement avec la pathogénie au cours des années 1880 et 1890. Mais si le pasteurisme propose un réel modèle pathogénique, la reconnaissance du caractère héréditaire général des maladies nerveuses relève $d^{\prime}$ une théorie, voire d'une idéologie médicale. La révolution pathogénique qui a lieu néanmoins a nécessité la reconnaissance d'éléments morbides constants et de leurs processus de genèse, définis à l'intérieur de cadres physiologiques théoriques, reposant sur l'expérimentation animale, applicables à la pathologie. Ces processus ne sont pas décrits comme des mécanismes physiologiques, mais apparaissent plutôt relever de déterminismes pathologiques propres, le pathologique devenant, selon la conception de Canguilhem, une espèce du normal ${ }^{102}$ et un état possible de la vie ${ }^{103}$. C'est en ce sens que la pathogénie devient, ainsi que Charcot le précise dans la leçon d'ouverture de sa nouvelle chaire en $1882^{104}$, I'un des domaines privilégiés de la nosologie, c'est-à-dire du domaine de réforme des opérations d'individualisation des maladies par la distinction des éléments lésés, de leurs mécanismes d'apparition, dans leurs conditions pathologiques et spécifiques d'apparition.

\section{Un cadre théorique fin de siècle : La théorie du neurone}

\section{La théorie du neurone à la Salpêtrière}

Si le terme neurone est dû à Heinrich Wilhelm Gottfried Waldeyer-Hartz (1836-1921 $\left.{ }^{105}\right)$, I'aventure officielle du concept débute au congrès de la Société anatomique allemande, où Ramón y Cajal présente pour la première fois ses préparations microscopiques suscitant un vif intérêt chez le plus célébre des

102 - Canguilhem, op. cit. in n. 13.

103 - Claude Debru, Le Possible et les biotechnologies (Paris : Hermann, 2003).

104 - Jean-Martin Charcot, Leçons sur les maladies du système nerveux faites à la Salpêtrière (Paris : Delahaye, 1887), t. III, leçon d'ouverture.

105 - Heinrich Wilhelm Gottfried Waldeyer-Hartz, Ueber einige neuere Forschungen im Gebiete der Anatomie des Centralnervensystems, Deutsche medicinische Wochenschrift, 17 (1891), 1213-1218, 1244-1246, 1287-1289, 1331-1332, 1350-1356. 
Jean-Gaël BARBARA

histologistes allemands, Rudolf Albert von Kölliker (1817$\left.1905^{106}\right)$. Ce dernier découvre ainsi que la réaction noire de Golgi peut être contrôlée et utilisée avec systématicité. La diffusion de ce type de recherche suit des cours sinueux ${ }^{107}$ jusqu'en France, où il parvient à la faculté de médecine et dans les laboratoires d'hôpitaux. Le médecin belge Arthur Van Gehuchten (1861-1914), professeur d'anatomie et de neurologie à l'université catholique de Louvain, présent au congrès de Berlin, reproduit rapidement certains résultats et les publie dans la revue francophone de Louvain, La Cellule, dès $1890^{108}$. L'année suivante, le docteur français Léon Azoulay entreprend les premières recherches systématiques, dans notre pays, par la méthode de Golgi ${ }^{109}$. Elles donnent lieu en 1894 à une longue série de publications sur du matériel humain ${ }^{110}$. La technique de Golgi est en réalité connue depuis longtemps en France, mais aussi en Allemagne ${ }^{111}$. Dès 1875, Ranvier vante ses mérites, mais il met également en garde ses utilisateurs contre ses caprices, dans ses leçons réalisées à partir de $1872^{112}$. Ranvier a pu enseigner la technique au Collège de France et en assurer la diffusion en Europe. Aussi, avant 1895, le laboratoire de Dejerine est un autre haut lieu de propagation de la méthode de Golgi. $\mathrm{M}^{\mathrm{me}}$ Dejerine, qui a suivi les cours de Ranvier, y mène ses 106 - Santiago Ramón y Cajal, Recollections of my life (Cambridge : MIT Press, 1989), 355-358.

107 - Les travaux de Cajal sont d’abord reproduits par Kölliker, Pedro Ramón, Mihály Lenhossék, Gustaf Magus Retzius, Sala, Ludwig Edinger, en Espagne, Allemagne, Italie, Suède et Belgique. Voir Arthur Van Gehuchten, Préface du $1^{\mathrm{er}}$ octobre 1893 à la première édition de I'Anatomie du système nerveux de l'Homme, leçons professées à l'université de Louvain (Louvain : Uystpruyst-Dieudonné, 1897).

108 - Arthur Van Gehuchten, Contribution à l'étude de la muqueuse olfactive chez les mammifères, La Cellule, 6 (1890), 395-407. Id., La structure des centres nerveux : La moelle épinière et le cervelet, La Cellule, 7 (1891), 1-44. D’autres études par la méthode de Golgi parurent les années suivantes dans la même revue.

109 - Mathias Duval, Préface de l'ouvrage de Santiago Ramón y Cajal, Les Nouvelles idées sur la structure du système nerveux chez l'Homme et les Vertébrés (Paris: Reinwald, 1895), xi-xii.

110 - Azoulay publie notamment une série d'articles sur ce sujet dans les Comptes rendus de la Société de Biologie, en 1894, dont: Particularités de la structure du cervelet chez l'enfant (211); Structure de la corne d'Ammon chez l'enfant (212); Aspects des cellules névrogliques dans les organes nerveux cérébraux de l'enfant (225); Méthodes de Golgi modifiées (300) ; Bipolarité des cellules des ganglions rachidiens chez le foetus humain (collatérales commissurales des veines postérieures pour la colonne de Clarke) (404); Méthodes de Golgi (remarques, confirmation par la méthode de Cox) (419.

111 - Jean-Gaël Barbara, Santiago Ramón y Cajal (1852-1934) et la France, Lettre des neurosciences, 33 (2007), 3-5.

112 - Louis Ranvier, Traité technique d'histologie (Paris : Savy, 1875). 
Relations médecine - sciences...

recherches avec des élèves comme le jeune médecin, André Thomas, qui a vérifié les résultats de Cajal sur le cortex ${ }^{113}$. Mais un élève de Mathias Duval (1844-1907), le docteur Cyprien Conil, devance ces travaux avec la publication, dès 1892, d'un mémoire sur l'étude du bulbe olfactif par la méthode de Golgi ${ }^{114}$. Dans le domaine de l'enseignement médical, Mathias Duval et Edmond Retterer diffusent à partir de la même année les travaux de Cajal à la faculté de médecine de Paris, comme le fera Adrien Charpy à Toulouse ${ }^{115}$. Parmi ces personnalités, Azoulay, Duval, Dejerine et Jean Nageotte (1866-1948 ${ }^{116}$ ), deviennent tous des amis de Cajal et des défenseurs fidèles de la théorie du neurone, lorsqu'elle est ultérieurement remise en cause, notamment dans certains milieux médicaux ${ }^{117}$. Les deux grandes séries de travaux les plus spectaculaires alors sont les études polémiques de Duval, étayant sa doctrine de I'amiboïsme des neurones ${ }^{118}$, et celles incontestées des Dejerine, qui aboutissent à l'œuvre monumentale d'anatomie nerveuse, encore célébrée, l'Anatomie des centres nerveux (1895 $\left.{ }^{119}\right)$.

Il est incontestable que les milieux médicaux français et la Société de Biologie ont joué un grand rôle dans la réception de la théorie du neurone, alors que Ranvier, au Collège de France, et les professeurs en Sorbonne restent en dehors de la polémique, ou attaquent vivement le concept de neurone ${ }^{120}$. Les raisons de cette acceptation par les médecins sont multiples et complexes; elles semblent toucher aux bénéfices mutuels pro-

113 - André Thomas, Contribution à l'étude du développement des cellules de l'écorce cérébrale par la méthode de Golgi, Comptes rendus de la Société de Biologie (1894), 66-70. Javier DeFelipe, Edward Jones, Cajal on the cerebral cortex (NewYork: Oxford University Press, 1988). Edward Jones, Plasticity and neuroplasticity, Journal of history of the neurosciences, 9 (2000), 37-39.

114 - Cyprien Conil, Bulbe olfactif : Étude par la méthode de Golgi, Mémoires de la Société de Biologie (1892), 179-190.

115 - Duval, op. cit. in n. 109, xi.

116 - Chef des travaux anatomiques des maladies du système nerveux à la Salpêtrière de 1894 à 1896.

117 - Joseph Grasset, Neuropathologie : Grandeur et décadence du neurone, L'Année psychologique, 10 (1903), 260-283. Joseph Grasset (1849-1918) est un médecin de Montpellier qui publie plusieurs traités sur les maladies du système nerveux de 1876 aux années 1910. Voir aussi Javier DeFelipe, Edward Jones, Cajal's degeneration and regeneration of the nervous system (New York: Oxford University Press, 1991).

118 - Jules Soury, L'Amiboïsme des cellules nerveuses, critique des théories édifiées sur cette doctrine (Paris: Naud, 1901).

119 - Jules Dejerine, Anatomie des centres nerveux (Paris : Rueff, 1895).

120 - Jean-Gaël Barbara, The physiological construction of the neurone concept (18911952), Comptes rendus biologies, 329 (2006), 437-449. 
Jean-Gaël BARBARA

curés par l'interaction entre une nouvelle doctrine scientifique et l'évolution de la clinique et de l'anatomopathologie des maladies du système nerveux. Ce qu'il faut comprendre avant tout, c'est ce qu'apporte initialement la théorie à la médecine.

\section{L'unification des pathogénies cellulaire et wallérienne par la théorie du neurone}

La médecine a, depuis l'accueil de la pathologie de Virchow, une vision cellulaire des phénomènes morbides, des dégénérescences des tissus, en considérant les déterminismes locaux dans I'extension des lésions des cellules ${ }^{121}$, bien que son cadre philosophique se soit émancipé d'une vision trop restrictive de la maladie décrite seulement par une typologie des atteintes cellulaires. L'association étroite de la clinique et de l'anatomopathologie est fondée sur le concept de cellule, par des corrélations fines établies à l'intérieur de cadres institués par les modèles pathogéniques. C'est la voie de la pathologie préconisée, par exemple, dans I'Essai de pathogénie, où Ernest Coulon (1868 - ?) souhaite « ramener l'étude de toute la pathologie à un problème de biologie cellulaire ${ }^{122} »$.

En 1892, I'année même où Duval incorpore dans son enseignement la doctrine du neurone, il publie la septième édition de son célèbre Cours de physiologie, où il présente encore le tissu nerveux comme étant constitué de deux éléments, les fibres et les cellules ${ }^{123}$. Cette dichotomie dans les éléments nerveux rappelle les archaïsmes des travaux de Charles-Philippe Robin (1821-1885), ce professeur d'histologie qui refusait la généralité de l'état cellulaire en demeurant en faveur de la fibre, entité tout aussi autonome, selon lui, morphologiquement et fonctionnellement. Dans le domaine de I'histologie du tissu nerveux, la théorie cellulaire n'est pas complètement effective.

Les travaux démontrant la continuité des fibres et des cellules, leur dépendance trophique ou les liaisons de leurs phénomènes de dégénérescence constituent pourtant autant de façons d'aborder la question centrale de leurs relations réelles, sans

121 - Mais aussi les atteintes fonctionnelles globales comprises comme dérèglements généralisés du système nerveux.

122 - Ernest Coulon, Considérations sur la nature de la paralysie générale (Paris : Charaire, 1896), chap. III.

123 - Mathias Duval, Cours de physiologie (Paris : Baillière, 1892). 
Relations médecine - sciences...

qu'aucun consensus n'émerge. Au début des années 1890, le problème n'est pas résolu et prend une tournure très polémique en neurologie. Dejerine se place comme défenseur de la position périphériste en reconnaissant que, contrairement au modèle wallérien, bon nombre de pathologies débutent par une lésion primitive périphérique. II affirme même qu'une lésion de l'axone (cylindre axe) "retentit sur la cellule d'origine ${ }^{124}$ ». Une certaine confusion règne entre lésions des cellules et lésions des fibres, tant sont nombreux les modèles de dégénérescence: ascendant, descendant, rétrograde, orthograde, wallérien, non wallérien, etc. La pathogénie classique semble en déroute.

C'est dans ce contexte que la théorie du neurone apparaît chez nombre de médecins comme une heureuse simplification, alors qu'elle est jugée sans intérêt par certains anatomistes et physiologistes, à l'instar d'Albert Dastre (1844-1917), en Sorbonne ${ }^{125}$. Elle est d'abord, selon le mot de Coulon, " la restitution à la cellule nerveuse de son entité biologique que sa complexité de structure, son étrangeté apparente de développement et sa difficulté d'observation avaient [...] laissé ignorée des observateurs ${ }^{126} »$. Mais elle est surtout un cadre simplificateur et c'est Raymond, le successeur de Charcot, qui exprime le mieux cette idée. "[...] en pathologie nerveuse on a le tort d'abuser des entités morbides, on a le tort de conférer à de simples syndromes une autonomie qui leur fait défaut, en leur assignant des lignes de démarcation absolument arbitraires ${ }^{127}$. " Cette sentence est placée en préliminaire à la question de nosologie de la paralysie ascendante aiguë, cette pathologie que nous avons déjà mentionnée au sujet des travaux de Dejerine visant à déceler une lésion centrale, alors que seule des atteintes des racines antérieures sont décelées. La pathogénie de cette affection se situe en effet dans le cadre de la problématique entre périphéristes et centralistes. Mais pour Raymond, si les lésions des cellules et des fibres dans une région ou une autre sont variables, n'y a-t-il pas lieu de considérer, plutôt que la constance d'un ordre chronologique et spatial arbitraire des lésions, une régularité topologique plus générale ? Or, c'est ce qu'offre la théorie du neurone en permettant de localiser un ensemble de lésions de cel-

124 - Grasset, op. cit. in n. 117, 266

125 - Barbara, op. cit. in n. 120.

126 - Coulon, op. cit. in n. 122, 68.

127 - Fulgence Raymond, Leçons sur les maladies du système nerveux (Paris : Doin, 1897), $2^{\mathrm{e}}$ série, 41. 
Jean-Gaël BARBARA

lules et de fibres appartenant à une même unité physiologique et pathologique, un neurone particulier. Qu'importe finalement dans un premier temps si la dégénérescence débute dans la cellule ou la fibre, si l'on peut reconnaître d'abord que ces lésions sont localisées dans un neurone unique. "C'est vous dire, conclut Raymond, qu'il y a lieu aussi de renoncer à cette sorte d'opposition qu'on admettait jadis entre les cellules nerveuses et les fibres nerveuses: cellule et fibre nerveuse ne font plus qu'un, celle-ci étant le prolongement cylindre axile, par conséquent la continuation directe de celle-là ${ }^{128}$. » La pathologie constitue donc un domaine scientifique privilégié, dans lequel la théorie du neurone est attendue, pour éliminer une dichotomie cellule/fibre caractéristique de ce que nous avons dénommé le paradigme nerveux, demeuré en réalité beaucoup plus longtemps prégnant en physiologie pendant plusieurs décennies du XX $x^{\mathrm{e}}$ siècle ${ }^{129}$.

\section{Théorie du neurone, nosographie et anatomie normale}

Le concept de neurone permet à l'anatomie et à la pathologie du système nerveux de construire un nouvel ordre histologique tridimensionnel se superposant aux ordres anciens bidimensionnels des faisceaux et des couches de cellules. L'organisation des tissus ne se décrit plus en cellules d'origine et faisceaux efférents ou afférents, mais en chaînes de neurones, en systèmes neuroniques, dont l'extension englobe un espace dendritique de réception et un espace axonique d'émission de l'influx nerveux. Cette idée ne peut être plus clairement illustrée que par I'opposition entre I'histologie ancienne de la rétine (celle de Duval par exemple ${ }^{130}$ ) et la nouvelle histologie neuronale de Cajal ${ }^{131}$.

Dans le domaine de la nosographie, cette mise en ordre des éléments neuroniques a des conséquences heureuses puisqu'elle permet à des médecins comme Raymond de proposer de nouvelles façons de décrire la pathogénie. L'ancienne a pris modèle sur

128 - Raymond, op. cit. in n. 127, 42.

129 - Jean-Gaël Barbara, "La constitution d'un objet biologique au xx siècle : Enquête épistémologique et historique sur les modes d'objectivation du neurone », thèse de doctorat (univ. Paris 7, 2007).

130 - Mathias Duval, Structure et usages de la rétine, thèse pour le concours d'agrégation anatomie et physiologie (Paris: Baillière, 1872).

131 - Voir références et analyses in Barbara, op. cit. in n. 129. Santiago Ramón y Cajal, Histologie du système nerveux de l'Homme et des Vertébrés (Paris: Maloine, 1909-1911). 
Relations médecine - sciences...

les anciennes conceptions étiologiques en réfléchissant sur les interactions entre pathologies, à savoir si l'une pouvait être la cause de l'autre. Raymond propose désormais de considérer plusieurs pathologies ensemble, pour comprendre si l'une n'est pas une forme semblable $d^{\prime}$ une autre, c'est-à-dire affectant un même neurone, mais dans des conditions sensiblement différentes. II s'agit en réalité d'une nouvelle façon d'aborder les rapports entre pathologies - comme ceux existant entre la paralysie ascendante aiguë, les poliomyélites antérieures et la polynévrite motrice -, qui a véritablement valeur de programme.

" Il appartient aux recherches de l'avenir de nous éclairer, poursuit Raymond, sur la subordination de ces syndromes à la nature, à la durée et au mode d'application des agents infectieux ou toxiques que nous soupçonnons de les engendrer. II leur appartient aussi de mettre en lumière l'influence que la qualité, la dose, la durée d'application de l'agent pathogène sont susceptibles d'exercer sur la nature et la localisation des altérations subies par les neurones moteurs ${ }^{132}$. »

C'est la voie que suit également Dejerine, en prenant une part plus active dans la construction même de la théorie. Car si le neurone permet de regrouper dans un même lieu des lésions caractéristiques de pathologies proches, la diversité des pathologies et des lésions peut inversement indiquer de nouveaux neurones. C'est donc ici la pathologie qui informe l'anatomie. L'œuvre des Dejerine, I'Anatomie des centres nerveux, est le résultat de cette démarche systématique par l'utilisation de coupes microscopiques et macroscopiques sériées et par la technique de dégénérescence des fibres améliorée par Vittorio Marchi (1851-1908). La mise au point d'une grande platine mobile ${ }^{133}$ sur un microscope fabriqué par Nachet \& fils permet de suivre les faisceaux de dégénération secondaire jusqu'aux cellules d'origine - ce qui n'était pas possible avec la technique de Golgi ${ }^{134}$ - pour mettre en évidence de nouveaux systèmes de

132 - Raymond, op. cit. in n. 127, 45.

133 - Jules Dejerine, Sur un nouveau microscope à grand champ de vision pour les explorations méthodiques des grandes surfaces, Comptes rendus de la Société de Biologie (1895), 411-412.

134 - « La méthode [de Golgi] ne permet le plus souvent de saisir que la cellule d'origine ou l'arborisation terminale, ou le cylindraxe dans son trajet intermédiaire, de sorte qu'elle est le plus souvent impuissante à nous renseigner si telle cellule ou tel noyau est en rapport avec telle autre ou tel noyau. » (André Thomas, Le Cervelet : Étude anatomique, clinique et physiologique (Paris: Steinheil, 1897), 151.) 
Jean-Gaël BARBARA

neurones ${ }^{135}$. La théorie du neurone trouve là un renfort solide, soutenu par des techniques différentes de celles de son élaboration, adaptées à un matériel humain, dans une perspective clinique large, réellement utile.

\section{Biologie du neurone et nouvelle pathologie expérimentale}

Ce type d'unification des données cliniques et expérimentales, autour d'un même objet - le neurone - sert de base commune pour interpréter les grandes fonctions comme le sommeil, la mémoire, ou I'attention ${ }^{136}$. À l'opposé de ces domaines largement spéculatifs, et non sans relations avec eux, se développe une biologie cellulaire du neurone. Ce nouvel objet est étudié comme un lieu où s'exerce l'effet des intoxications, des infections, ou encore de l'hérédité ${ }^{137}$, fondant une étiologie proprement neuronale. L'ancienne dichotomie cellule-fibre (c'est-à-dire celle entre le corps cellulaire du neurone et son axone, selon la nouvelle terminologie) devient une première forme de partition cellulaire du neurone, dans lequel on distingue deux pôles possédant différentes susceptibilités aux agents toxiques, et répondant à leurs attaques par des réactions concertées, qui font du neurone un système adaptatif vivant, possédant ses propres mécanismes de régulation, de défense. Dans cette perspective, le neurone est constitutif de systèmes cellulaires et devient lui-même un système de biologie cellulaire décomposable en parties.

Un second événement fondateur de cette biologie neuronale est la découverte en 1894 par Franz Nissl (1860-1919) de la coloration par le bleu de toluidine des " corps de Nissl", dont on saura plus tard qu'il s'agit de l'ergastoplasme (en fait des citernes de réticulum endoplasmique rugueux, associées aux ribosomes basophiles ${ }^{138}$ ). Pour ce médecin, en son temps, ce ne sont

135 - Comme ceux du pied du pédoncule cérébral. Jules Dejerine, Sur I'origine corticale et le trajet intra-cérébral des fibres de l'étage inférieur ou pied du pédoncule cérébral, Comptes rendus de la Société de Biologie (1893), 193-206. Voir en particulier la description des systèmes de neurones, 203, et la figure, 204. Franz Nissl, Ueber die Veranderungen der Ganglienzellen am Facialiskern des Kaninchens nach Ausreissung der Nerven, Allgemeine Zeitschrift für Psychiatrie, 48 (1892), 197.

136 - Léon Azoulay, Psychologie histologique et texture du système nerveux, L'Année psychologique, 2 (1895), 255-294.

137 - Ernest Coulon, Considérations sur la nature de la paralysie générale (Paris : Charaire, 1896), chap. III.

138 - Franz Nissl, Ueber die Veranderungen der Ganglienzellen am Facialiskern des Kaninchens nach Ausreissung der Nerven, Allgemeine Zeitschrift für Psychiatrie, 48 (1892), 197. 
Relations médecine - sciences...

là, dans le corps cellulaire des neurones, que de simples corpuscules bleus, se détachant sur le fond d'une matrice incolore. Il ne peut s'agir d'artefacts insignifiants, car leurs présence et absence sont corrélées avec l'état de santé du neurone. Leur disparition - dénommée chromatolyse - est un événement manifeste de certains états pathologiques et survient, par exemple, lorsque l'axone est sectionné ${ }^{139}$ ou lors de diverses intoxications ${ }^{140}$. Ces observations deviennent des données fondamentales dans la réévaluation de certaines distinctions nosologiques par l'analyse des modes de dégénérescence des cellules nerveuses, de l'axone au soma, ou du soma à l'axone, à l'intérieur d'un neurone ${ }^{141}$. C'est dans ce cadre d'études que se placent les travaux d'Alzheimer, qui collabore avec Nissl pour mettre en évidence les lésions neuronales associées aux démences ${ }^{142}$, et ceux de Georges Marinesco (1863-1938).

Chez ces auteurs, on peut dégager deux tendances dont I'une vise à différencier des pathologies sur la base de lésions spécifiques - ou d'ensembles, ou encore de successions, spécifiques de lésions - et l'autre regroupe par un même mécanisme pathogénique des maladies considérées comme des formes proches. Or, comme précédemment au sujet des fibres nerveuses, les modèles pathogéniques généraux, élaborés cette fois autour du concept de neurone par la pathologie expérimentale et I'histologie, trouvent dans la clinique une diversité d'observations qu'on cherche à subsumer sous de nouvelles entités pathologiques, malgré parfois de sévères contradictions. C'est là tout l'enjeu de nombreuses polémiques qui éclatent entre Raymond, Marinesco et Dejerine. Dejerine critique, par exemple, la réunion par Marinesco de la névrite interstitielle et de l'atrophie musculaire de type Charcot - Marie, dans un même schéma pathogénique ${ }^{143}$. Raymond n'est pas opposé à de tels rapprochements, en particulier concernant la réunion de la névrite et de la poliomyélite. "Depuis l'avènement de la doctrine du neurone, rappelle-t-il, cette conception nosologique a trouvé une 139 - Nissl, op. cit. in n. 138, 197.

140 - Georges Marinesco, Pathologie générale de la cellule nerveuse : Lésions secondaires et primaires, La Presse médicale, 5 (1897), 41.

141 - Fulgence Raymond, Leçons sur les maladies du système nerveux (Paris: Doin, 1897), $2^{\mathrm{e}}$ série, 293-295. Azoulay, op. cit. in n. 136.

142 - Voir Gzil, op. cit. in n. 24, pour I'histoire des recherches d'Alzheimer.

143 - Raymond, op. cit. in n. 141, 6e série, 209-210. 
Jean-Gaël BARBARA

base solide, objective, [...] Les récentes recherches de M. Marinesco et d'autres histologistes nous ont appris que l'état de souffrance de la fibre nerveuse retentit sur la structure de la cellule trophomotrice. Donc, s'il y a indépendance histologique, il n'y a pas indépendance au point de vue des affinités pathologiques ${ }^{144}$. » Raymond se fait donc souvent conciliant en montrant en quoi la pathologie expérimentale de Marinesco fournit des concepts et des mécanismes pathogéniques utiles, permettant des "localisations [des lésions qui] traduisent la souffrance $d^{\prime}$ un seul et même organe, le neurone ${ }^{145}$ ». Cependant, il resitue finalement la clinique au premier plan : " [...] pour nous, cliniciens, le côté anatomopathologique [...] est peu de chose, le côté pronostic est tout. Or, en l'espèce, le pronostic ne peut être fondé que sur une connaissance approfondie des modalités cliniques $d^{\prime}$ un processus qui a pour substratum un même organe, le neurone ${ }^{146}[\ldots]$. » Or, c'est précisément I'objet de nombreuses attaques de Dejerine qui dénonce la signification pathologique abusive de la chromatolyse rencontrée après autopsie chez de nombreux patients, sans symptômes associés ${ }^{147}$. C'est aussi ce qui pousse certains auteurs, comme Albert Gombault (1844-1904), à refuser de restreindre la pathologie à des formes uniquement neuronales ${ }^{148}$. Alors que d'autres, à I'instar de Jérôme-Maurice Gerest (1871-?), recherchent une synthèse entre théorie du neurone et clinique ${ }^{149}$.

144 - Raymond, op. cit. in n. 141, 4érie, 401-402 (c'est Raymond qui souligne).

145 - Raymond, op. cit. in n. 141, $2^{\mathrm{e}}$ série, 362-363 (c'est Raymond qui souligne).

146 - Raymond, op. cit. in n. 141, $2^{\mathrm{e}}$ série, 362-363.

147 - Jules Dejerine, Sur I'absence d'altérations des cellules nerveuses de la moelle épinière dans un cas de paralysie alcoolique en voie d'amélioration, Comptes rendus de la Société de Biologie (1897), 399-402. Id., Sur la chromatolyse de la cellule nerveuse au cours des infections avec hyperthermie, Comptes rendus de la Société de Biologie (1897), 728-729.

148 - « [La nouvelle conception neuronale du système nerveux] devrait même, d'après quelques auteurs, servir de base unique à toutes les études d'histologie, normale et pathologique, et il ne saurait plus être question que de neurones de divers ordres et de maladies de neurones. Mais son application nous paraît irréalisable dans la pratique ; en outre, elle serait loin de cadrer avec bien des faits observés en pathologie humaine. » (Victor-André Cornil et Louis Ranvier, Manuel d'histologie pathologique (Paris: Baillière, 1902), $5^{\mathrm{e}}$ éd.) Nous devons cette citation à François Clarac. Sur Gombault, voir : François Clarac et Bernard Lechevalier, Albert Gombault (1844-1904), un pionnier des neurosciences, Revue neurologique, 162 (2006), 253-263.

149 - Jérôme-Maurice Gerest, Les Affections nerveuses systématiques et la théorie des neurones (Paris : Baillière, 1898). 
Relations médecine - sciences...

La théorie du neurone constitue donc un cadre théorique qui permet $d^{\prime}$ interrompre certains conflits inextricables dans la recherche de pathogénies cellulaires, ou impliquant des fibres nerveuses, c'est-à-dire en éliminant les apories entre certains scénarios neurologiques concernant la mise en cause d'une lésion primitive, dont le caractère antécédent n'est plus le but ultime des recherches. Ce qui compte désormais, c'est d'identifier des maladies de certains neurones. L'étiologie, se fondant toujours à la fois sur les mécanismes pathogéniques et les entités morbides, trouve désormais dans le neurone une manière simplificatrice de les décliner, formant ultérieurement la base nouvelle de la sémiologie, telle que la constituera par exemple Dejerine ${ }^{150}$. Mais cette théorie donne lieu, et c'est une tendance humaine toujours observée, à des spéculations poussées trop avant, ainsi qu'à des modèles expérimentaux dont on use parfois aveuglément pour expliquer la physiologie et la pathologie. Quoi qu'il en soit, la pathogénie neuronale s'ouvre à cette époque en un champ de recherche fécond par ses techniques, ses concepts et ses succès indiscutables, d'une actualité toujours plus grande aujourd'hui, et I'on peut dire que les nouvelles idées sur le neurone et la connaissance de ses affections pathologiques constituent ensemble, dès cette époque, une véritable neurobiologie.

\section{Conclusion}

L'étude des procédés d'individualisation des maladies du système nerveux, à l'intérieur de cadres pathogéniques précis, permet de définir de nouveaux modes d'interaction entre la médecine et les sciences au $\mathrm{Xx}^{\mathrm{e}}$ siècle. Il s'agit d'interactions positives, où les modèles pathogéniques apparaissent comme des domaines de convergence d'idées et de données de la clinique et des sciences, par des jeux de légitimation réciproque. Ces modèles qu'on utilise souvent abusivement dans une première période, trouvent ensuite leur juste place dans la nosographie.

La distinction de facteurs externes et internes, consubstantiels, dans la détermination de l'évolution des champs de recherche médicale, se résout dans la mise en évidence de déterminants, plus ou moins généraux. Certains relevant des rapports entre la médecine et les sciences peuvent être mis en évidence par 150 - Jules Dejerine, Sémiologie des affections du système nerveux (Paris : Masson, 1914). 
I'analyse des contextes d'actualisation des rencontres heuristiques entre la médecine et les théories, pratiques et nouvelles disciplines scientifiques. Les milieux médicaux qui sont les théâtres de ces échanges conservent néanmoins une autonomie, garantie par la clinique, qui en font des territoires à la fois indépendants, favorables, ou parfois en contradiction avec les sciences. Ainsi, la clinique des maladies du système nerveux à la Salpêtrière a bénéficié des possibilités de rassembler des patientes atteintes de pathologies homogènes et diverses, laissées à l'étude de jeunes médecins acquis à la théorie cellulaire, la microscopie, en partie seulement à la pathologie expérimentale, dans des cadres théoriques larges et souples. La mise à distance contrôlée de la pathologie au sein des autres sciences et ses rapprochements avec l'anatomie et la physiologie, tels qu'ils pouvaient se pratiquer lors de discussions à la Société de Biologie, se sont développés dans des cadres philosophiques évolutifs, sans crainte de subordonner les processus morbides aux théories physiologiques et pathogéniques du moment.

Par de telles analyses épistémologiques, conservant un lien avec I'histoire sociale, les modèles pathogéniques paraissent constituer un véritable moteur à la fois dans la pathologie et les sciences, par des remises en cause réciproques et des effets d'entraînement dans l'adoption de nouveaux procédés techniques au sein des milieux hospitaliers. Cependant, I'approche scientifique, dont la visée recèle un degré plus important de généralité, permet le développement de théories (théorie du neurone) en coordonnant les modèles pathogéniques, en les rendant plus féconds et plus opérants en pathologie. La pathologie conserve toujours au final la liberté de juger si le chemin parcouru est d'intérêt en clinique, ou si la légitimité du domaine scientifique est dépassée. Dès lors, comme l'atteste chaque jour davantage la recherche biologique, la médecine, qui vise à déterminer les bases scientifiques des pathologies et des phénomènes pathogéniques élémentaires en vue de traitements, doit être considérée comme une activité à la fois autonome et liée de façon synergique avec la science, l'une formant I'extension de l'autre, et vice versa, ainsi que le réalisent, selon Canguilhem, le normal et le pathologique. Cependant, cette aventure humaine exceptionnelle, dans laquelle chaque malade a sa place, ne se concentre désormais pas tant sur la définition 
Relations médecine - sciences...

biologique des maladies, que sur leurs mécanismes fins, sur les traitements possibles et leurs conséquences sur la vie du malade, par un rationalisme biomédical qu'on souhaite toujours plus unifié, efficace et porteur d'espoir. 
Remerciements. Je tiens à remercier très chaleureusement Michel Fardeau pour m'avoir reçu à la Pitié-Salpêtrière et pour notre discussion au sujet de Dejerine, Jacques Poirier pour son jugement avisé et ses suggestions, Jean-Claude Dupont, François Clarac, ainsi que Chantal Barbara pour sa relecture.
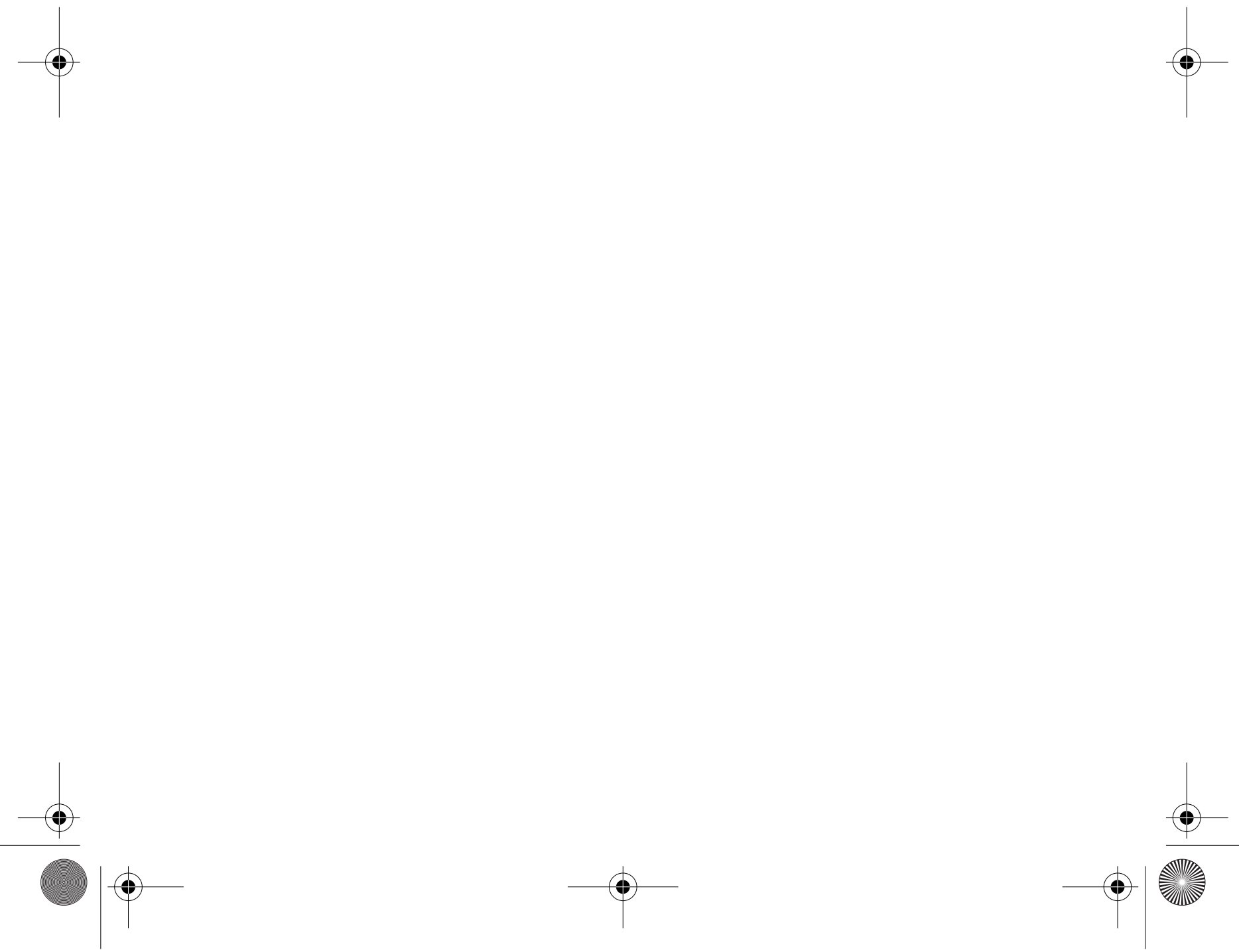